\title{
A systems-guided approach to discover the intracellular target of a novel evolution-drug lead
}

Sourav Chowdhury ${ }^{1}$, Daniel C. Zielinski ${ }^{2}$, Christopher Dalldorf ${ }^{2}$, Joao V Rodrigues ${ }^{1}$, Bernhard O. Palsson ${ }^{2,3}$, Eugene I Shakhnovich ${ }^{1 *}$

${ }^{1}$ Chemistry and Chemical Biology, Harvard University, Cambridge, MA, USA

${ }^{2}$ Department of Bioengineering, University of California, San Diego, La Jolla, CA, USA

${ }^{3}$ Novo Nordisk Foundation Center for Biosustainability, Technical University of Denmark, Kemitorvet, Building 220, 2800 Kongens Lyngby, Denmark

*Correspondence

\begin{abstract}
:
Understanding intracellular antibiotic targeting and the associated mechanisms leading to bacterial growth inhibition has been a difficult problem. Here, we discovered the additional intracellular targets of the novel "evolution-drug" lead CD15-3 designed to delay the emergence of antibiotic resistance by inhibiting bacterial DHFR and its Trimethoprim resistant variants. Overexpression of DHFR only partially rescued inhibition of E. coli growth by CD15.3 suggesting that CD15.3 also inhibits a non-DHFR target in the cell. We utilized untargeted global metabolomics and the metabolic network analysis along with structural similarity search of the putative targets to identify the additional target of CD15-3. We validated in vivo and in vitro that besides DHFR CD15-3 inhibits HPPK (folK), an essential protein upstream of DHFR in bacterial folate metabolism. This bivalent cellular targeting makes CD15-3 a promising lead to develop a "monotherapy analogue" of combination drugs.
\end{abstract}

\section{Introduction:}

Antibiotic molecules are designed to selectively interact with and inhibit the actions of essential bacterial proteins (Butler et al., 2013). However, even conventional antibiotic drugs are found to target multiple molecular targets inside bacterial cells, and hence demystification of antibiotic targeting inside cells presents a potential challenge (Silver, 2007). Recent advances in systems biology tools are making intracellular drug target analysis an increasingly accessible task (Rabinowitz et al., 2011). It is worth noting that even with well-documented antibiotics with known specific molecular targets, understanding the series of events leading to bacterial death has been a complex problem. The need for such understanding appears even more pressing as 
increasing numbers of novel antibiotic formulations are emerging to tackle the problem of drug resistance.

Bacteria deploy a panoply of strategies to evade drug actions, and the bacterial "resistome" is a tight assembly of multi-layered highly orchestrated mechanisms (Olivares Pacheco et al., 2013). In the current context of widespread antibiotic resistance, including the emergence of "multidrug resistant" ESKAPE variants, a mechanistic understanding of intracellular antibiotic targeting and what leads to bacterial death stands as an immensely pertinent problem.

In our previous study we presented a new compound, CD15-3, as an in-house developed "evolution-drug" lead that significantly delays the emergence of resistant phenotypes. CD15-3 was designed to interact with wild type DHFR (dihydrofolate reductase) and its trimethoprim (TMP) resistant mutants (Zhang et al., 2020). TMP is a well-known antifolate antibiotic that inhibits bacterial growth by selectively blocking bacterial DHFR - a critical enzyme in bacterial one-carbon metabolism. However as resistance against TMP evolves very rapidly, CD15-3 was designed to interact with TMP-resistant DHFR mutants, thereby blocking this evolutionary escape route. Our short-term evolution experiment showed that CD15-3 was able to prevent the emergence of most resistant phenotypes at the timescale of the evolutionary experiment. Most marginally resistant phenotypes which evolved at the end of a 30 day long run of adaptive evolution did not show any mutation associated with the target gene locus and was primarily due to duplication of the part of the genome containing genes of efflux machinery. Overexpression of folA, which codes of DHFR, was able to partially rescue CD15-3-induced growth inhibition. However, the lack of complete growth rescue from overexpression of DHFR potentially indicates the presence of an additional non-DHFR intracellular target of CD15-3. A potential bivalent property of CD15-3 would additionally make development of resistant phenotypes a much more difficult evolutionary journey.

For a long time, multi-targeting has been an alternative strategy to develop combination therapies and design treatment protocols to extend drug life. In this respect, CD15-3 is not just a lead "evolution-drug" which delays drug resistance but is also a potential "monotherapy analogue" of combination drugs targeting more than one molecular target.

In this work, we deployed an integrated multi-layered approach to hunt for the potential nonDHFR target of CD15-3 (Figure 1). We analysed untargeted global metabolomics to unravel differences in the global metabolome upon CD15-3 treatment and tried to capture potential hotspots of metabolic perturbation. We used metabolic supplementation experiments and recovery assays to identify which metabolic pathways are most perturbed upon CD15-3 treatment.

We then overexpressed the genes close to the points of metabolic perturbation and looked for growth recovery signals. We analysed these experimental data in the context of the metabolic network to narrow down the search options for the CD15-3 cellular target. Overexpression of shortlisted genes helped to identify the potential CD15-3 non-DHFR target, which was further confirmed by carrying out imaging experiments and enzyme assays. This work confirms the 
potential of CD15-3 with bivalent properties as a "evolution-drug" lead. Furthermore, the analysis pipeline we developed integrating multiple interconnected layers of cellular organization to identify the intracellular target can be potentially used to hunt for molecular targets for other novel biologically active compounds.
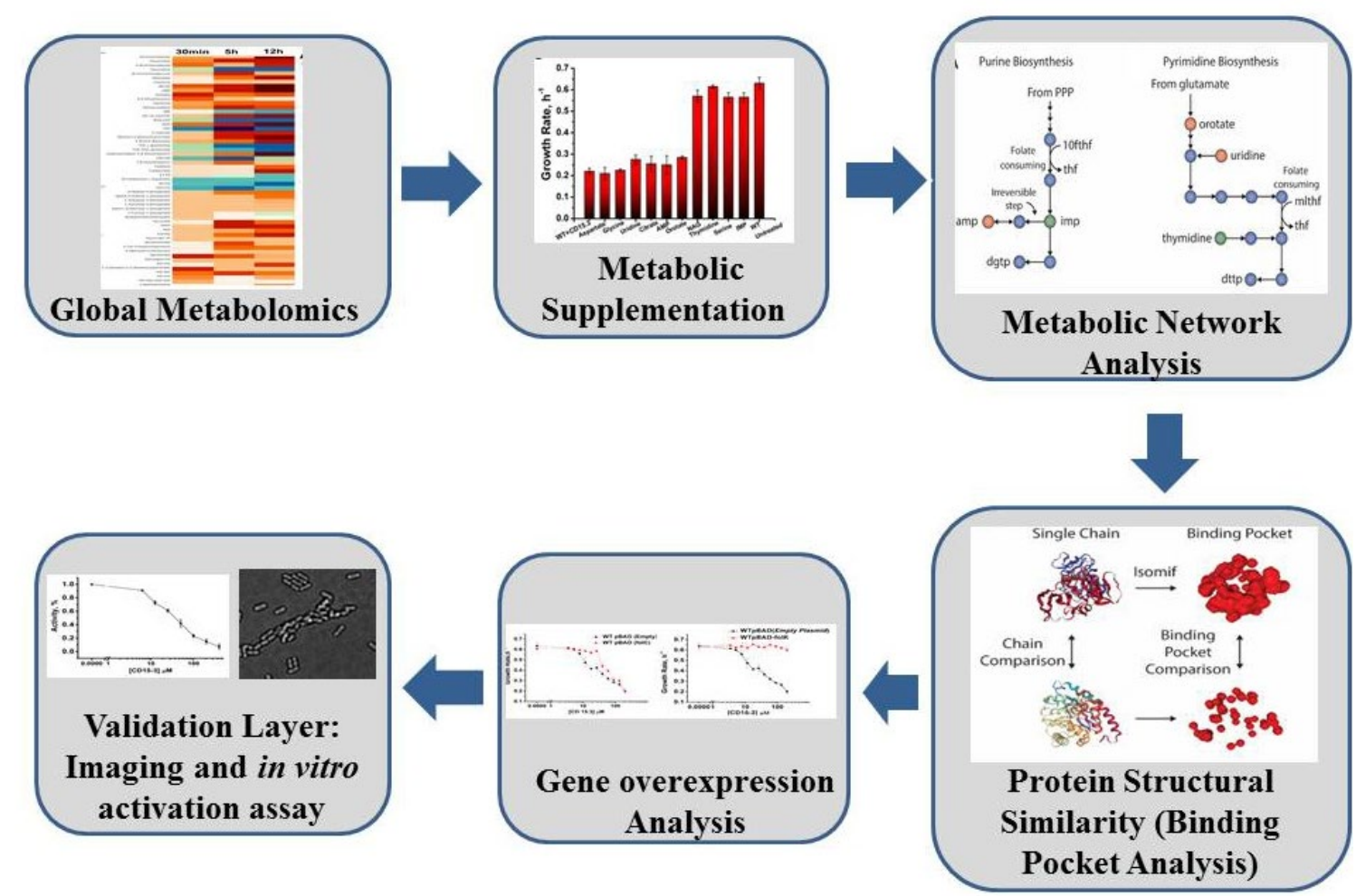

Figure 1: Schematic representation of the integrated workflow deployed to search for the non-DHFR intracellular target of CD15-3. Untargeted global metabolomics identified metabolites which showed abundance differences upon CD15-3 treatment. This primary systemwide information was used for understanding the nature of metabolic perturbation upon CD15-3 treatment and identifying the putative non-DHFR target of CD15-3. Information from each layer is used in the subsequent layer of analysis which helped in narrowing down the potential target options and finally identifying the non-DHFR target of CD15-3 


\section{$\underline{\text { Results: }}$}

Untargeted Global Metabolomics reveals CD15-3 induces metabolic stress with a strong impact on nucleotide metabolism

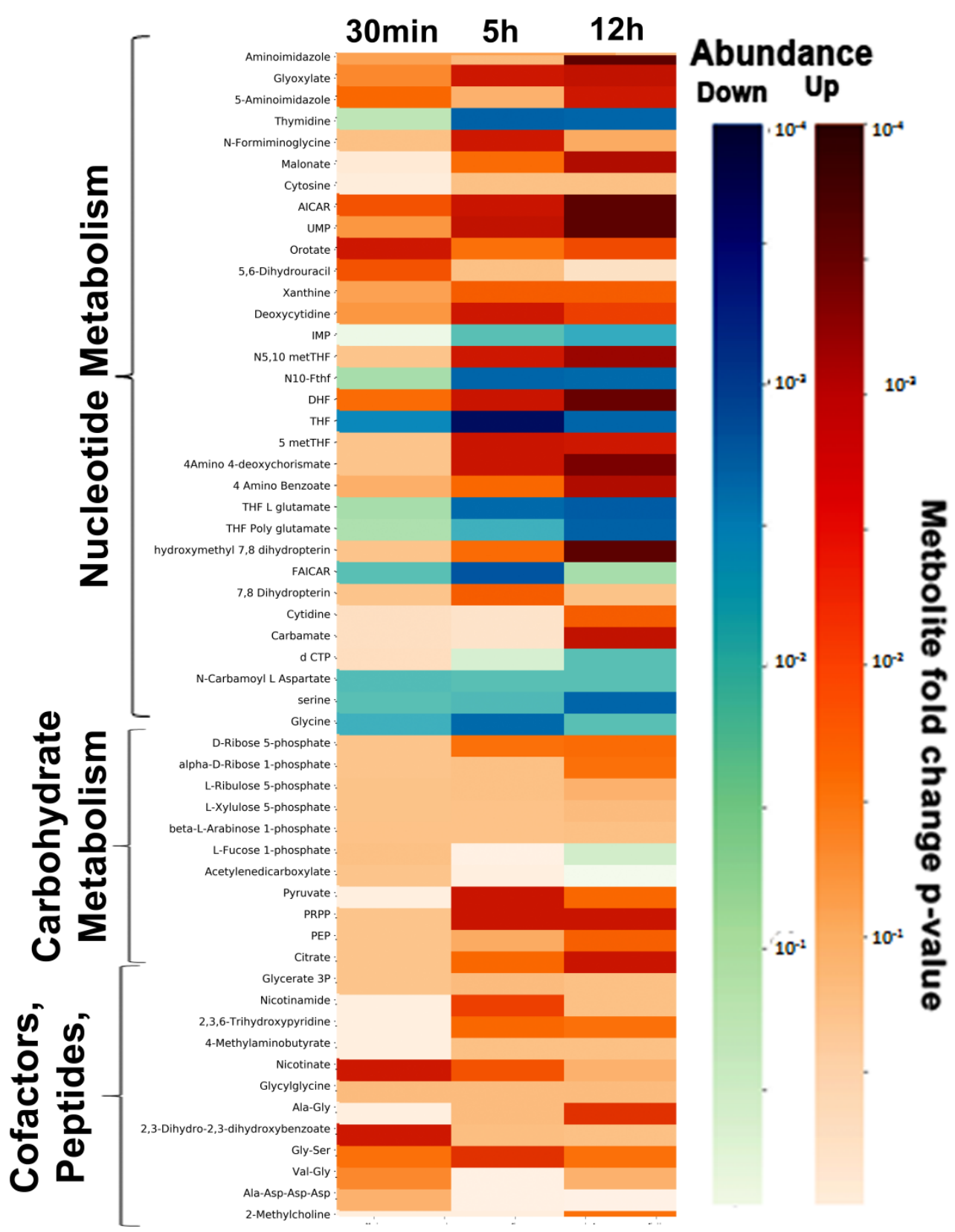

Figure 2. Major changes in the global metabolome under conditions of CD15-3 treatment. Heat map describing the global metabolomic profile of untreated (WT) and treated (WT+CD153) cells. Color gradient indicates fold differences (in log scale) observed in the metabolite abundance levels in the CD15-3 treated set compared to the untreated WT cells. Horizontal lines in the heat map indicate metabolites. Color bar shows negative logarithm of p-values (Mann- 
Whitney Test) associated with abundance ratios (treated vs. control) of each metabolite. A higher intensity of the blue bar indicates more depletion while a higher intensity of the orange bar indicates more accumulation.

In a previous study, we showed that cellular overexpression of DHFR helped cells to partially recover from CD15-3-induced growth inhibition (Zhang et al., 2020). As full rescue of growth at all concentrations of CD15-3 was not observed with overexpression of DHFR, we went on to explore possible non-DHFR intracellular targets of CD15-3.

We first measured the metabolic perturbations upon CD15-3 treatment to obtain a metabolomewide view of CD15-3 action inside the cell. To that end we carried out untargeted global metabolomics measurements to obtain the comparative global metabolome under untreated and CD15-3 treated conditions. Cells were grown in the presence and absence of CD15-3 for different lengths of time and were harvested for processing. Comparative metabolite abundances showed progressively increasing differences in cellular metabolism as the cells were exposed to CD15-3 for longer lengths of time. Figure 2 shows the differences reflected in metabolites involved in nucleotide metabolism, carbohydrate metabolism, cofactors, and peptides. As observed, a five hour exposure to CD15-3 significantly impacts the global metabolism of cells, with even higher differences being observed at 12 hours exposure.

A

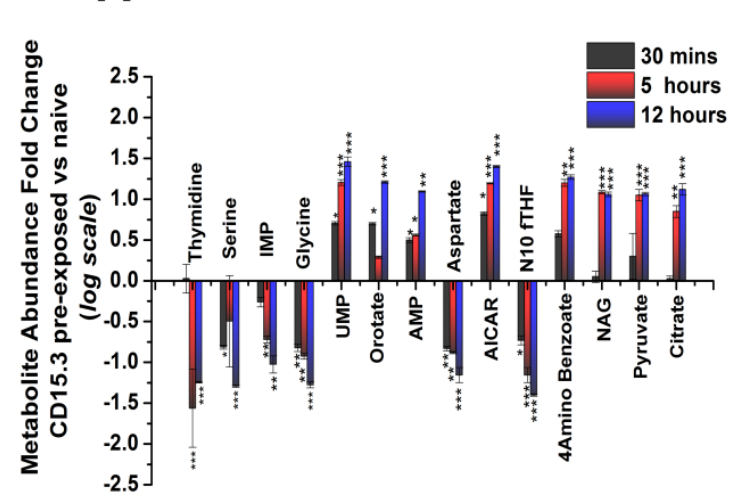

C

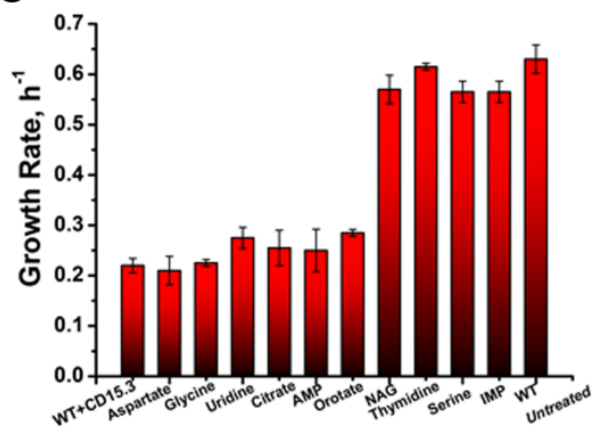

B

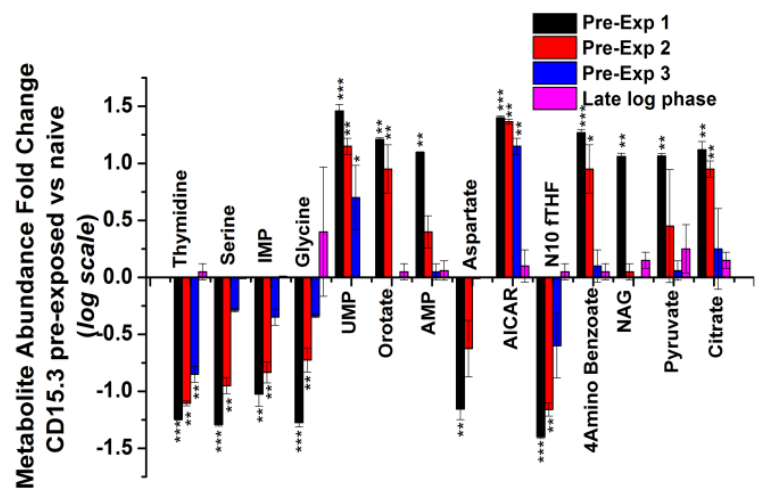

D

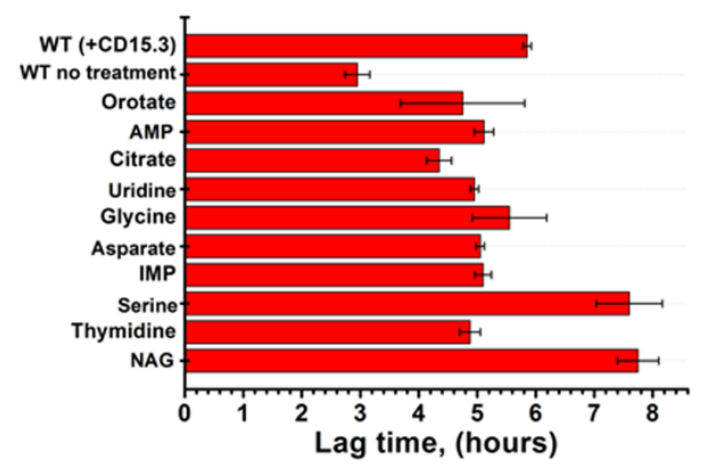


Figure 3: Representative metabolites of nucleotide metabolism show significant fold changes upon CD15-3 treatment. (A) Bar plot showing abundance fold differences (log scale) of selected metabolites as the cells grow in the presence of CD15-3. Metabolomics data for the treated and untreated control cells were gathered by harvesting cells at three different time points as shown in the figure. (B) Bar plot showing the metabolite abundance profile (log scale) of selected metabolites under the condition of the recovery experiment when cells pre-incubated with CD15-3 were grown in normal M9 media supplemented with $0.8 \mathrm{~g} / \mathrm{L}$ glucose. The fold differences in the metabolite abundances are the ratios of metabolite abundance observed in the (recovering) pre-exposed/CD15-3 treated cells to that of the naïve cells. (* indicates metabolite abundance ratios with $\mathrm{p}$ values $\leq 0.05, * * \leq 0.001, * * * \leq 0.0001$ as derived from a Mann-Whitney test). (C) Bar plot showing how metabolite supplementation impacts growth rate in the presence of CD15-3. Rescue from CD15-3-induced growth inhibition is observed with thymidine, serine, NAG and IMP supplementations. A fixed concentration of $200 \mu \mathrm{M}$ CD15-3 was used in these supplementation experiments. (D) Bar plot showing variation in lag-time during metabolite supplementation experiments. NAG and serine supplementation leads to a prolonged lag time compared to other metabolites.

Thymidine, a constituent of the pyrimidine biosynthesis pathway shows a 15-fold drop in abundance after 5 hours of growth with CD15-3 treatment and a 17-fold drop after 12 hours of growth (Figure 3A). 4-aminobenzoate which participates in folate metabolism and a precursor to THF biosynthesis shows 15-fold higher abundance at 5 hours of growth and 18-fold higher abundance at 12 hours of growth with CD15-3 treatment (Figure 3A). 4-aminobenzoate is synthesized from chorismate and is an important metabolic intermediate which leads to the biosynthesis of a host of crucial metabolites such as folates. N10-formyl-THF, a precursor in the THF biosynthesis, showed 12-fold up regulation at 5 hours of growth with CD15-3 treatment and 15-fold higher abundance after 12 hours growth (Figure 2B). Folates are crucial for the biosynthesis of many important cellular metabolites, including glycine, methionine, formylmethionine, thymidylate, pantothenate and purine nucleotides. Our comparative global metabolome showed significant fold differences in many of these metabolites indicating some plausible perturbation around the folate pathway and gross perturbation distributed throughout the overall nucleotide metabolism. For example, serine shows 11 -fold lower abundance at 5 hours of growth and almost 20-fold lower abundance Iat 12 hours of growth with CD15-3 treatment (Figure3A). Likewise glycine also showed continuous cellular depletion upon CD15-3 treatment with 25-fold lower abundance after 12 hours of growth with CD15-3 treatment (Figure3A). Another interesting metabolic marker for perturbed purine metabolism is AICAR. AICAR is located upstream to serine and glycine biosynthesis steps and showed almost 8-fold higher abundance at 30 minutes of growth and 16-fold higher abundance at 5 hours of growth (Figure3A). Cellular buildup of AICAR at early stages of treatment indicates that purine metabolism gets disrupted quite early under CD15-3 treatment. UMP, a constituent of pyrimidine metabolism also showed cellular build up with 32-fold higher abundance after 12 hours of growth with treatment. Also, significant fold differences in the abundance levels of various peptides, co-factors and lipids were observed which too could be attributed to a CD15-3 induced 
metabolic stress response (Zhang et al., 2020). We observed significant fold differences in some metabolites constituting carbohydrate metabolism. Pyruvate and citrate cellular build-up has been known to be metabolic stress response (Shimizu, 2014). Under CD15-3 treatment we observed 11 fold higher abundance of pyruvate and 12 fold higher abundance of citrate at 12 hours growth with CD15-3 treatment (Figure3A). Cellular buildup of citrate under CD15-3 treatment potentially indicates a possible slowdown of glycolysis and in turn energy metabolism.

\section{Recovery experiments reveal the connection of metabolite perturbations to CD15-3 response}

We went on to determine the abundance levels of the relevant metabolites during recovery following CD15-3 treatment. To determine, which metabolites display the most delayed recovery after CD15-3 treatment and hence were most impacted upon the treatment, we incubated WT cells in CD15-3 for 12 hours and subsequently transferred them to normal M9 media supplemented with $0.8 \mathrm{~g} / \mathrm{L}$ glucose. In a parallel control, WT cells were grown for 12 hours in M9 media and subsequently regrown in normal M9 media. Metabolite abundance levels were measured at four discrete time points (Supplementary Figure 1) of cell harvesting. Notably, AICAR and several other metabolites that showed significant abundance changes in early stages of treatment gradually restored to normal levels during recovery (Figure3B). However, thymidine, IMP, and serine were found to have significant fold differences until the preexponential phase 3. A similar trend was observed with AICAR, UMP, and N10 formyl THF, and these metabolites took a longer time to recover. $\mathrm{N}$-acetylglutamate (NAG), which is a constituent of the ornithine biosynthesis pathway via the formation of N-acetyl ornithine, was found to have significantly higher abundance upon treatment and was found to respond much earlier in the recovery experiment with abundance levels returning to normal. NAG build up also plausibly indicates some perturbation in the pyrimidine metabolism as ornithine participates in the biosynthesis of arginine, which interacts with pyrimidine metabolism through sharing carbamoyl phosphate as a precursor.

Metabolite abundance data and recovery experiments indicated multiple perturbations in nucleotide metabolism. This is consistent with the folate metabolism as intended target of CD153 , with folate derivatives being a key cofactor in nucleotide biosynthesis. In our previous report we showed that CD15-3 interacts with DHFR and any impact on cellular DHFR would affect cellular tetrahydrofolate titer further perturbing protein and nucleotide biosynthesis (Rodrigues and Shakhnovich, 2019; Zhang et al., 2020). Further, this perturbation in nucleotide metabolism as captured by the global metabolome could suggest that CD15-3 primarily disrupts nucleotide metabolism. 


\section{Metabolic supplementation reveals a pathway-specific rescue from CD15-3 growth inhibition}

The metabolite response indicates broad perturbations to metabolism across many distinct pathways. To narrow down which perturbations are most directly linked to CD15-3-dependent growth inhibition, we chose to use a subset of the identified metabolic markers for metabolic supplementation experiments to attempt a rescue from CD15-3-induced growth inhibition. To this end, wild type cells were grown in the presence of the externally supplemented metabolites under conditions of CD15-3 treatment.

In all these experiments, the external metabolite supplement concentration was kept at $0.5 \mathrm{mM}$. Control experiments were performed in the absence of CD15-3 treatment to check for intrinsic toxicity of these external metabolite supplementations (Supplementary Figure 2A). We observed that the metabolites selected for the metabolic supplementation experiment did not show toxic effect on the bacterial growth.

Also, we checked the possibility that these compounds might serve as alternate carbon sources. To that end we grew cells without CD15-3 treatment in M9 minimal media without glucose. The growth profile was compared with bacterial growth in M9 minimal media with $0.8 \mathrm{~g} / \mathrm{L}$ glucose, which is the typical media composition used in the rest of the study. We observed that, apart from thymidine, no other metabolite served as an alternate carbon source (Supplementary Figure 2B). In the presence of thymidine, cells did show slow growth with a very long lag phase compared with the glucose control.

Supplementation with thymidine, NAG, serine and IMP showed growth rescue, as reflected in improved growth rates (Figure 3C) under the conditions of CD15-3 treatment. On the other hand, negligible or no effect on the growth rates were observed with external supplementation of aspartate, glycine, uridine, citrate, orotate and AMP. Thymidine and other metabolites which showed significant rescue of CD15-3 inhibition improved the growth rate to around $0.6 \mathrm{~h}^{-1}$ which is comparable to the growth rates observed in the WT cells in the absence of CD15-3.

It is interesting to note that metabolites whose external supplementation rescued growth rates did not significantly affect the lag time. Lag time in bacterial growth is a critical indicator of cellular adaptation in the ambient growing condition (Fridman et al., 2014). In our experimental condition a higher lag time in the presence of some external supplements and CD15-3 treatment reflects time of metabolic rewiring and adaptation in the supplement enriched growing condition. It is interesting to note that NAG and serine significantly prolonged the lag time (Figure 3D) under treatment conditions although both have shown to have positive impact in improving growth rates (Figure.1C). Thus, NAG and serine could be considered as partial rescuers from CD15-3 induced stress with improvement on only growth rate and worsening the lag time. 

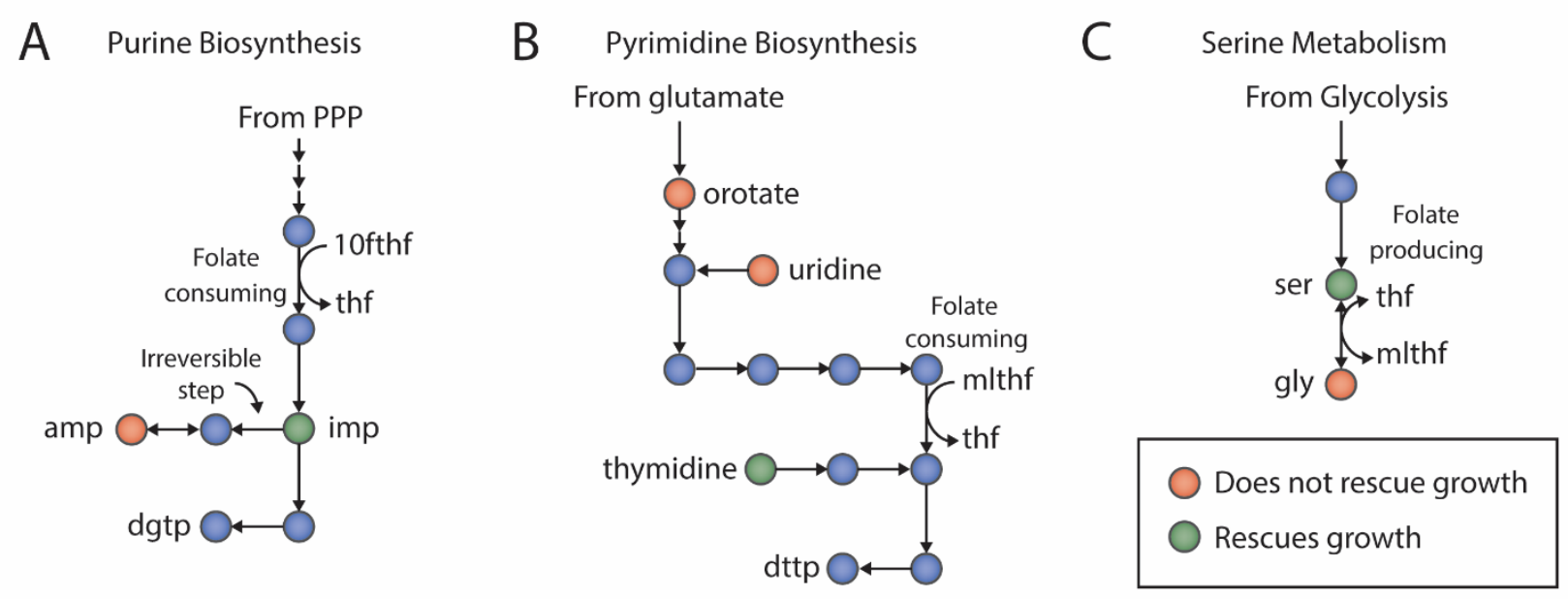

Figure 4 Effect of nutrient supplements on CD15-3-treated cells indicate broad inhibition of available folate. A) Purine biosynthesis pathway. Both IMP and AMP supplements are downstream of folate-requiring steps. However, AMP is unable to be converted to dGTP due to an irreversible step in the pathway, while IMP can be converted to both dATP and dGTP. B) Pyrimidine biosynthesis pathway. The growth rescuing supplement, thymidine, enters the pathway downstream of the folate requiring step. Supplements that enter upstream of the folate requiring step, namely orotate and uridine, did not rescue growth. C) Serine and glycine biosynthesis. Serine, which produces the charged folate form 5,10-methylene tetrahydrofolate when converted to glycine, rescued growth, while glycine itself did not rescue growth.

\section{Metabolic pathway analysis of nutrient supplement rescue experiments}

Metabolic supplementation demonstrated that diverse compounds were able to rescue inhibition by CD15-3, while other metabolites had no effect. To better understand the metabolic rationale for the supplementation rescue patterns of CD15-3, we utilized the most updated metabolic network reconstruction of E. coli, iML1515(Monk et al., 2017). We examined the trends in metabolite supplementation rescue experiments with respect to their location in corresponding metabolic pathways. We observed that the effectiveness of the supplement on rescuing growth was determined by the position of the supplement in the metabolic network vis a vis folate metabolism. Specifically, supplements that have the potential to mitigate folate deficiency, namely thymidine, IMP, and serine, were effective at rescuing growth. Thymidine and IMP bypass the folate-dependent step in pyrimidine and purine biosynthesis, respectively (Figure 4 $\mathrm{A}, \mathrm{B}$ ), while serine can contribute to folate production through its conversion to glycine (Figure 4C). Meanwhile, supplements that do not mitigate a folate deficiency do not rescue growth, even though these metabolites may be adjacent in the network to successful supplements, such as uridine, AMP, and glycine. Uridine is a pyrimidine precursor that is upstream of the folatedependent biosynthetic step, while glycine has an unclear role, as metabolism through the glycine cleavage chain produces folate, while conversion to serine consumes folate. Thus, although DHFR may not be the primary target of CD15-3, the inhibitory activity of CD15-3 still appears to primarily work through folate limitation. 


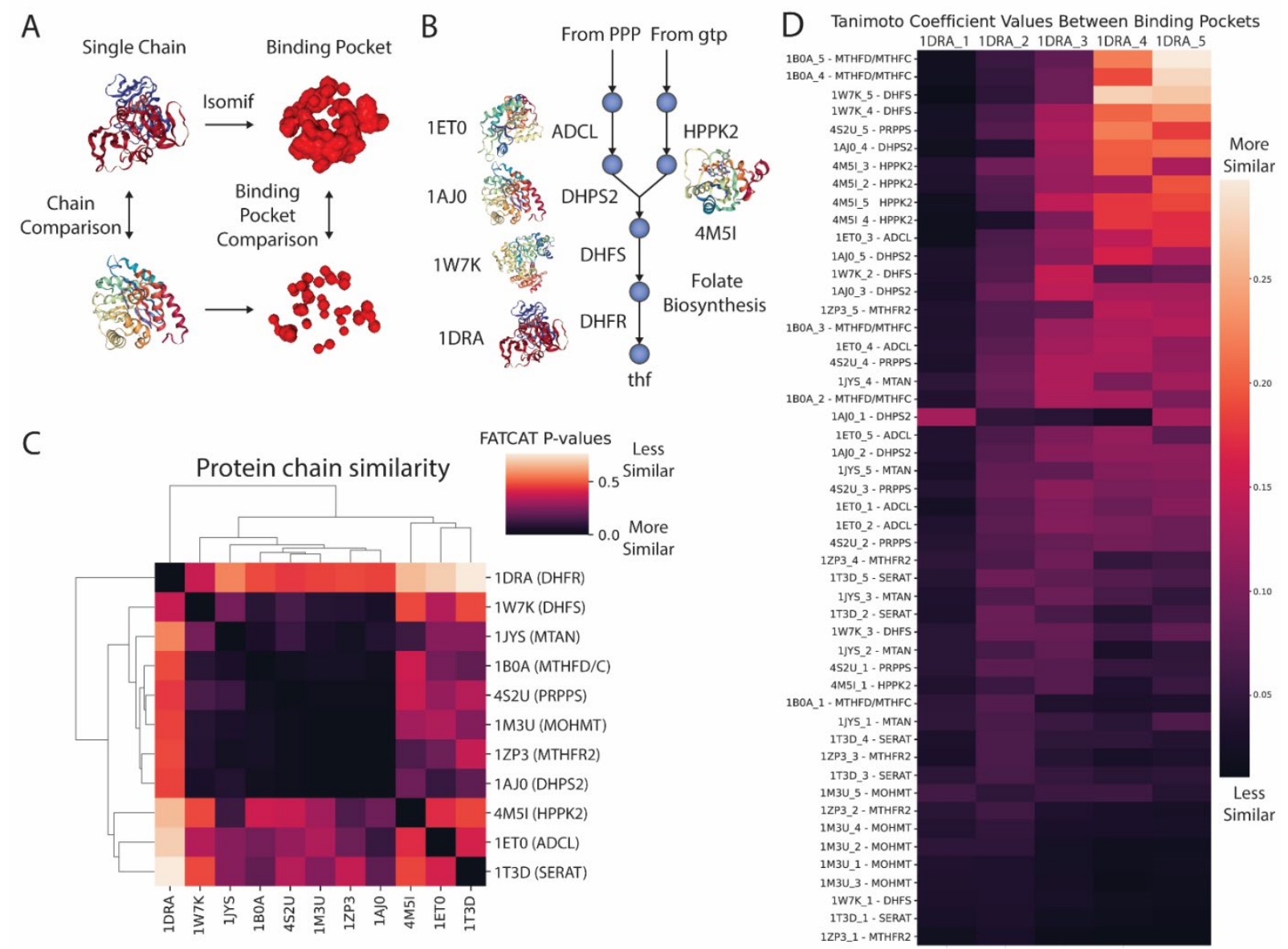

Figure 5: Protein structural similarity evidence supports other folate enzymes as possible targets of CD15-3. A) Overview of workflow for structural analysis. B) Overview of folate pathway and protein structures extracted from PDB. C) Results for whole structure similarity analysis utilizing FATCAT. Low p-values reported by FATCAT indicate more similar structures. D) Results for active site analysis using isomif for comparison. Protein structure identifiers are taken from PDB.

\section{Structural analysis of possible alternate binding targets}

Experimental evidence from metabolomics, supplementation, all suggested that folate perturbation is the primary mode of action of CD15-3. Thus, we hypothesized that the alternate target of CD15-3 also lies within the same metabolic pathway as DHFR. Notably, other enzymes in the folate biosynthetic pathway are also known to be drug targets, such as Dihydropteroate synthase, the target of sulfamethoxazole. To narrow down likely alternative targets of CD15-3, we employed a genome-scale reconstruction of enzyme protein structures, termed the GEM-PRO or Genome-scale Model with Protein Structures of E. coli (Monk et al., 2017) (Figure 5A, Figure 
5B). Utilizing the FATCAT algorithm for structural alignment ( $\mathrm{Li}$ et al., 2020), we found that DHFR did not have a high degree of structural similarity to any particular enzyme in the folate pathway based on whole chain alignment (Figure5C). However, protein structure analysis comparing the binding pocket of the intended target DHFR and other enzymes in the folate biosynthetic pathway via Tanimoto coefficients suggested alternative binding targets for CD15-3 (Figure 5D, Methods). Notable proteins with high binding pocket homology scores included several enzymes involved in folate biosynthesis and folate interconversion, such as MTHFC, HPPK2, and DHPS. Thus, we decided to screen several upstream enzymes in the folate biosynthetic pathway whose binding pockets have high homology to DHFR's. To that end we evaluated whether overexpression of any of these enzymes rescues growth inhibition by CD15.3

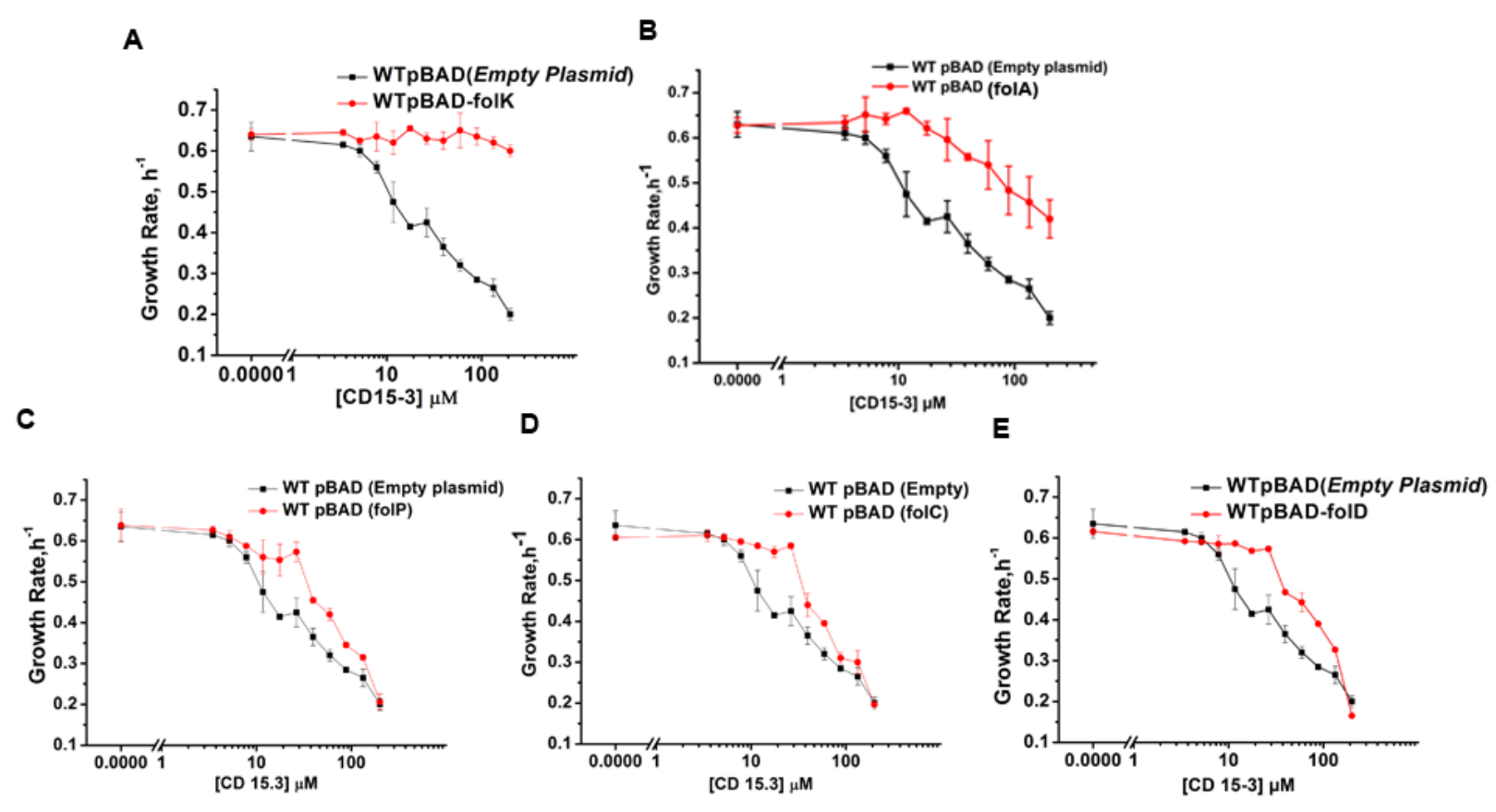

Figure 6: Overexpression of folk (encoding HPPK) was found to recover cells from CD15-3 induced growth inhibition. (A) Overexpression was induced with $0.1 \%$ arabinose and folk was expressed under $\mathrm{pBAD}$ promoter. Recovery of growth rate was found to occur at all the CD15-3 working concentration. (B) Overexpression of folA (encoding DHFR) was induced at $0.005 \%$ arabinose. folA overexpression was found to have partial effect in recovering growth rate in CD15-3 treated cells. Overexpression of (C) folP (encoding DHPS) (D) folC (encoding DHFS) and (E) fol D (encoding MTHFC) showed slight improvement in the growth rates of the CD15-3 treated cells only at lower concentrations $(<50 \mu \mathrm{M})$ of CD15-3. folP, folC and folD genes were overexpressed at $0.1 \%$ arabinose induction under $\mathrm{pBAD}$ promoter. 


\section{Gene overexpression experiments validate functional role of perturbed metabolic pathways in CD15-3 mechanism of action.}

Regulated overexpression of candidate genes viz., folK, folP, folC, folD, metF and pabC using the $\mathrm{pBAD}$ promoter with $0.1 \%$ arabinose induction was carried out to determine whether any of the overexpressed genes show recovery from CD15-3 induced growth inhibition and thus may be the non-DHFR target of CD15-3. In our previous study (Zhang et al., 2020) we showed that overexpression of folA (encoding DHFR) partially rescued CD15.3 induced toxicity at lower concentrations of CD15.3. However folA overexpression from $\mathrm{pBAD}$ was restricted to $0.005 \%$ arabinose induction to avoid overexpression induced toxicity (Bhattacharyya et al., 2016) which would complicate interpretation of the rescue experiments.

Of the assessed proteins besides folA (shown here for consistency on Fig.6A and (Zhang et al., 2020)) only overexpression of folK showed clear sign of rescue from growth inhibition. folK encodes for 6-Hydroxymethyl-7,8-dihydropterin pyrophosphokinase (HPPK). HPPK is a key enzyme in the folate biosynthesis pathway catalyzing the pyrophosphoryl transfer from ATP to 6-hydroxymethyl-7,8-dihydropterin, is an attractive target for developing novel antimicrobial agents (Bermingham and Derrick, 2002; Chhabra et al., 2013; Chhabra et al., 2012). Upon folK overexpression cells did not show any change in growth rates in a broad range of concentrations of CD15-3 (Figure 6A). It was found to be around $0.6 \mathrm{~h}^{-1}$ at all the concentrations of CD15-3, which is the typical growth rate of WT cell growing in the absence of CD15-3. folA overexpression (encoding DHFR) on the other hand with $0.005 \%$ arabinose induction showed partial recovery from CD15-3 inhibition (Figure 6B). Rescue in growth rate was more visible at lower CD15-3 concentration regimes.

We further over-expressed folP (encoding DHPS) (Figure 6C), folC (encoding DHFS) (Figure $5 \mathrm{E}$ ) and folD (encoding MTHFC) (Figure 6D) to see any possible rescue effect on growth rates. Only at lower concentrations of CD15-3 $(<50 \mu \mathrm{M})$ overexpression of these genes partially rescued growth inhibition. Overexpression of folD (Figure 6E) showed slight improvement in growth rate at mid concentration of CD15-3 (around $70 \mu \mathrm{M}$ ). E. coli folD gene encodes for a bifunctional enzyme having both methylenetetrahydrofolate dehydrogenase and methenyltetrahydrofolate cyclohydrolase activities. The dehydrogenase and cyclohydrolase reversibly catalyze oxidation of N5,N10-methylenetetrahydrofolate to N5,N10methenyltetrahydrofolate and the hydrolysis of N5,N10-methenyltetrahydrofolate to N10formyltetrahydrofolate and play critical role in purine metabolism.

We overexpressed two more genes and found no recovery effect. The metF gene encodes for methylene THF-reductase (MTHFR). Overexpression of metF did not show any rescue in CD153 induced growth inhibition (Supplementary Figure 3A). Next, we screened for ADCL which is encoded by gene pabC. pabC encoding aminodeoxychorismate lyase is involved in the biosynthesis of p-aminobenzoate (PABA) which is a precursor of tetrahydrofolate. ADCL converts 4-amino-4-deoxychorismate into 4-aminobenzoate (PABA) and pyruvate. Overexpression of pabC did not show any recovery of growth rates in CD15-3 treated cells (Supplementary Figure 3B). 
In comparison to the overexpression of the other candidate genes, folK overexpression showed a clear rescue effect, with growth rates staying around 0.6 hour $^{-1}$ even at the higher concentrations of CD15-3. This indicates strongly that CD15-3 interacts with cellular HPPK as its non-DHFR molecular target. The complete growth recovery observed with folK overexpression indicates that the growth-limiting folate perturbation originates in the folK mediated step, which in turn impacts rest of the folate pathway. In the Supporting Text (Supporting Information) we have provided an explanation as to why folA overexpression leads only to partial rescue while overexpression of folK resulted in full recovery from CD15-3 induced inhibition. In short, the reason for the difference is in different expression levels of folA and folK in overexpression experiment. The Supporting Text presented quantitative analysis of the effects of inhibition of two proteins by a common inhibitor and the competing factors of inhibition of both enzymes and sequestration of the inhibitor by an overexpressed enzyme. Supplementary Figure 4 summarizes the effects of various metabolic supplements used and gene overexpression strategies deployed and show how CD15-3 has a folate-related mechanism of action.

\section{Morphological changes induced by CD15-3 treatment are greatly relieved upon folK over- expression.}

Perturbation in the folate pathway leads to cellular filamentation and concomitant morphological changes (Ahmad et al., 1998; Bershtein et al., 2015; Bhattacharyya et al., 2021; Justice et al., 2008; Sangurdekar et al., 2011; Zaritsky et al., 2006). CD15-3 treated, and untreated WT cells were grown for 4 hours and subjected to DIC imaging. CD15-3 treated cells (Figure 7B) showed a considerable extent of cellular filamentation as compared to untreated WT (Figure 7A) cells grown for the same length of time.

A similar experiment was also done with WT cells overexpressing folk under pBAD promoter with $0.1 \%$ arabinose induction. WT cell sets overexpressing folK were grown for 4 hours under control and CD15-3 treatment conditions. WT cells overexpressing folK did not show any visible change in cellular shape and size (Figure 7D) compared to the untreated control (Figure 7C). Upon comparison of the median cell lengths (Figure 7E), a slightly higher median cell length was observed in the folK overexpressing cells with CD15-3 treatment (median cell length = $1.252 \mu \mathrm{m}$ ), as compared to cells under control conditions (median cell length $=1.06 \mu \mathrm{m}$ ). This slightly higher median cell length could be attributed to the fact that CD15-3 also targets cellular DHFR. Overexpression of folk although in majority nullifies the effects of CD15-3 on cell shape; a little change in median cell length still happens and could be potentially attributed to the interaction between CD15-3 and cellular DHFR. The overall pronounced rescue in cell length upon overexpressing folK further supports the conclusion that HPPK is the non-DHFR cellular target of CD15-3. 
$\mathbf{A}$

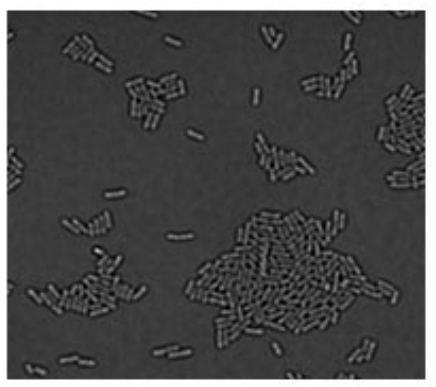

$\mathbf{C}$

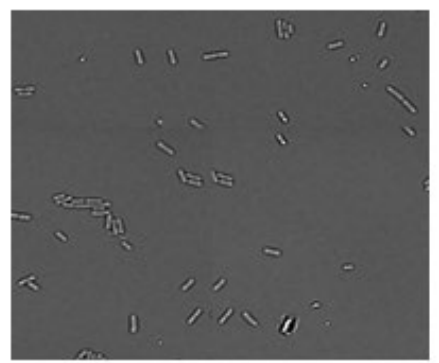

$\mathbf{E}$

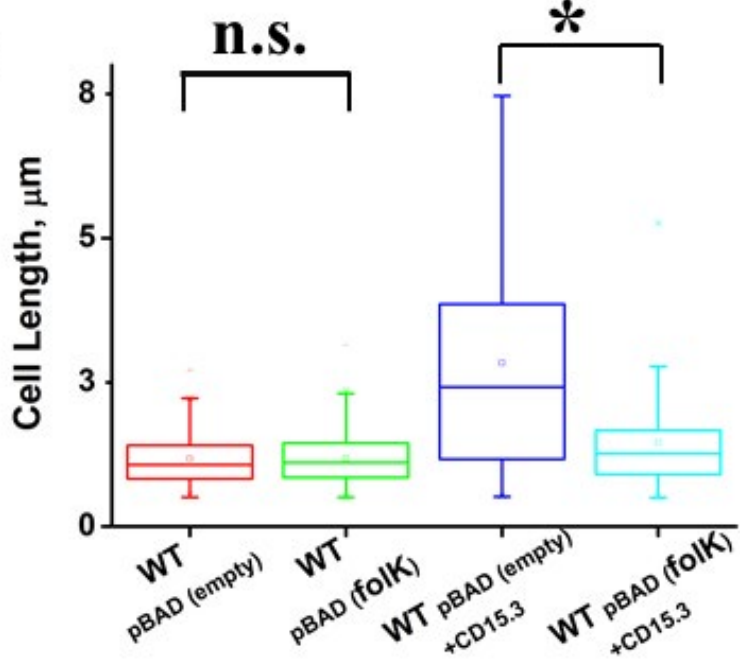

$\mathbf{B}$

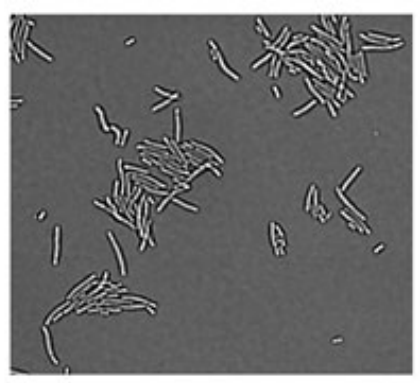

D

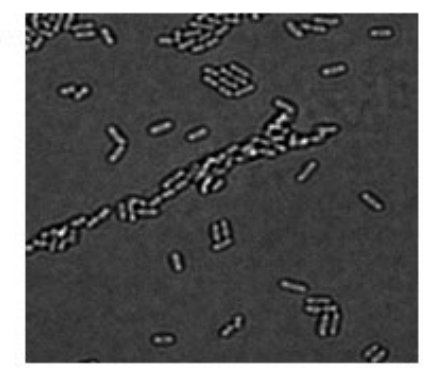

Figure 7: Overexpression of folK rescues cells from CD15-3 induced morphological changes. DIC image of WT cells under (A) control (no CD15-3 treatment) and (B) treated (CD15-3 treatment) conditions. CD15-3 treated cells shows visible signs of cellular filamentation. (C) DIC image of WT E. coli cells overexpressing folK under control (no CD15-3 treatment) and (D) CD15-3 treated condition. Unlike WT cells (B) showing cellular filamentation in presence of CD15-3, folk overexpressing cells upon CD15-3 treatment did not manifest visible signs of filamentation. (E) Distribution of cell lengths as derived from DIC imaging of WT and WT cells overexpressing folK under control and CD15-3 treatment conditions. Untreated WT and cells overexpressing folK had comparable cell lengths with median cell lengths of $1.06 \mu \mathrm{m}$. (n.s. indicates the distribution of cell lengths were not significantly different, Mann-Whitney test, $\mathrm{p}$-value $=0.952$ ). Both WT and cells overexpressing folK were subjected to CD15-3 treatment at IC 50 concentrations. WT treated cells were observed to have a filamentous morphology and the median cell length $(2.41 \mu \mathrm{m})$ double of that of the 
untreated WT set. WT cells overexpressing folk after CD15-3 treatment had a median cell length of $1.252 \mu \mathrm{m}$ which is slightly higher than that of untreated set. However, the cell size distribution of the cells overexpressing folK showed much less change after CD15-3 treatment compared to that observed for the WT (* indicates the distribution of cell lengths were significantly different, Mann-Whitney test, p-value $<0.001)$.
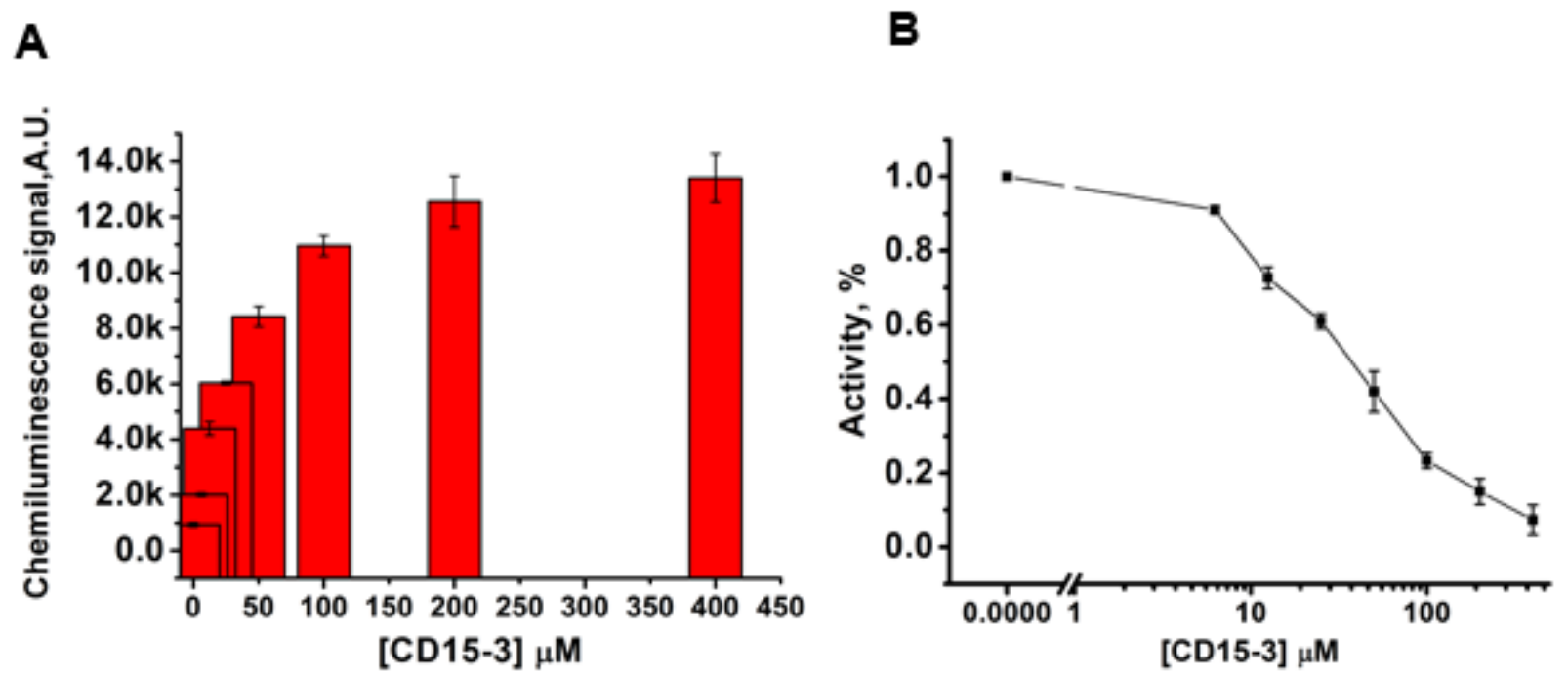

Figure 8: CD15-3 interacts with HPPK and inhibits its activity as is evident from the accumulation of ATP in the assay buffer, with a concomitant rise in the chemiluminescence signal. (A) Bar plot shows the chemiluminescence signals at various concentrations of CD15-3 in the HPPK assay buffer. As chemiluminescence signals depends on ATP titre in the assay buffer, HPPK which catalyses an ATP dependent reaction (0 $\mu \mathrm{M}$ CD15-3 in our study), upon catalytic activity leads to a drop in the chemiluminescence signal. Plot shows that with increasing CD15-3 concentrations, absolute chemiluminescence signal considerably enhances. This shows that CD15-3 inhibits HPPK activity leading to higher availability of unused ATP in the assay buffer, which in turn leads to higher chemiluminescence. (B) \% Activity was calculated (details of the relation mentioned in the results section) using the absolute chemiluminescence intensity values. 50\% activity ( $\mathrm{IC}_{50}$ ) was observed at $36.46 \mu \mathrm{M}$.

\section{In vitro HPPK activity assay reveals that CD15-3 inhibits HPPK encoded by folK}

Next, we aimed to verify in an in vitro assay that CD15-3 indeed inhibits HPPK in a concentration dependent manner.

To that end we performed a KinaseGlo ${ }^{\mathrm{TM}}$ assay to test for HPPK activity and its probable inhibition in presence of CD15-3. We derived HPPK by 1000 fold dilution of the cell lysates overexpressing HPPK under pBAD promoter at $0.1 \%$ arabinose induction. KinaseGlo ${ }^{\mathrm{TM}}$ assay is based on chemiluminescence (Tanega et al., 2009). With higher ATP concentration in the assay buffer, luciferase leads to the conversion of beetle luciferin to oxy-luciferin with the emission of light. The HPPK mediated reaction utilizes ATP leading to ATP depletion and hence drop in the 
chemiluminescence signal. Any potential inhibition of HPPK would retain original concentration of ATP keeping the chemiluminescence signal intact or like the control (with no HPPK activity). We observed a marked drop in the absolute chemiluminescence signal intensity in the HPPK reaction set with no CD15-3 in the assay buffer (Figure 8A). Interestingly presence of CD15-3 led to enhanced absolute chemiluminescence signal intensity (Figure 8A) suggesting that CD153 does inhibit HPPK. The inhibitory effect appeared to be progressively higher upon increase of the CD15-3 concentration in the reaction-assay-buffer. For control we performed similar experiments with $400 \mu \mathrm{M}$ of CD15-3 with one ATP dependent protein Adk and one ATP independent protein BSA to validate that the drop in signal we observe in presence of CD15-3 in the HPPK reaction-assay set is due to the specific inhibitory interaction between CD15-3 and HPPK (Supplementary Figure 5). We did not observe any drop in luminescence with BSA or ADK reaction set in the presence of CD15-3.

In our assay protocol, HPPK reaction was initiated with the introduction of the substrate, 6Hydroxymethyl-7,8-dihydropterin in the assay buffer (Supplementary Figure 5). We used the absolute chemiluminescence intensity values to calculate $\%$ activity using the following relation:

$$
\% \text { Activity }=\frac{\text { Signal at inhibitor concentration }- \text { Signal at no reaction }}{\text { Signal at zero inhibitor concentration }- \text { signal at no reaction }} \times 100
$$

where signal at inhibitor concentration is the chemiluminescence signal at any given concentration of CD15-3, signal at no reaction is the optical signal obtained when the substrate is not added and the reaction is not initiated.

Using this relation and plotting the absolute signal intensity values observed across the CD15-3 concentration gradient we found $50 \%$ activity ( $\mathrm{IC}_{50}$ ) observed at $36.46 \mu \mathrm{M}$ (Figure 8B).

\section{Discussion}

Antibiotic molecules are designed to interact with molecular targets which are essential to bacterial life processes. Although the design phase involves much precision to specifically interact with the specific target, under many conditions the designed molecule happens to interact with more than one cellular target (Silver, 2007). Many natural antibiotics including penicillin and macrolides are known to interact with more than one cellular target. Further, simultaneous targeting of more than one cellular target poses a challenge to evolving bacterial cells by blocking their evolutionary escape routes (Tyers and Wright, 2019). This minimizes the chances of emergence of resistant cells. This forms one of the foundations behind the current thrust and interest towards the development and use of combination-drug therapies(Sullivan et al., 2020). But a systematic understanding of the cellular targeting is of immense importance in antibiotic drug discovery programs as it provides a mechanistic insight into the drug action inside the bacterial cells. As antibiotic molecules target proteins which in turn constitute critical points in the bacterial metabolic scheme, an investigation of the metabolic architecture provides essential clues tracing the potential points of metabolic perturbations under conditions of antibiotic action. Critical analysis of such points of metabolic perturbation and its comparison with untreated control sets helps unveiling the antibiotic targets inside the bacterial cells. Recent 
advances in untargeted metabolomics have provided valuable insights into the global metabolome and helped to quantitatively identify the metabolic cascades impacted by perturbations (Vincent et al., 2016; Wu et al., 2015; Zampieri et al., 2017).

In the present study we deployed global metabolomics, gene over-expression, and growth recovery studies analysed in the context of the metabolic network to search for the cellular target of a novel "anti-evolution" lead CD15-3. We observed that CD15-3 markedly delays the emergence of antibiotic resistant bacterial phenotypes (Zhang et al, 2020). This could be attributed to factors like; 1) CD15-3 was designed to interact with WT as well as TMP resistant mutants which arise under conditions of TMP selection. Thus CD15-3 potentially blocks the evolutionary escape routes which $E$. coli traverses under TMP selection. It is to be noted that TMP is a classical antibiotic which disrupts bacterial purine metabolism by interacting with bacterial DHFR (Huovinen, 1987). 2) As CD15-3 has a non DHFR target too, thus blocking two cellular targets makes evolution of antibiotic resistant $E$. coli phenotypes more difficult. We hypothesized that CD15-3 is an interesting lead towards the development of the evolution drugs as "anti-evolution" strategies to address the problem of antibiotic resistance. The resistance which was observed in our 30-day long evolution experiment was not associated with any point mutation in a gene locus (Zhang et al., 2020). Rather it was duplication of part of the genome encoding generic efflux machinery with multiple pumps and transporters brought about a modest ( $\sim 2$ fold) increase of IC50 for CD15-3. Thus, it was important to determine an alternative target of CD15-3 in E. coli cells to better understand the mechanism of action of this prototypic "evolution-drug" inside bacterial cells.

Upon comparing the global metabolome of the CD15-3 treated cells with that of the untreated control and analysing the metabolite-abundance fold differences we identified metabolic cascades which are primarily perturbed by CD15-3.

As CD15-3 interacts with WT and TMP resistant mutants of DHFR, a possible multi-targeting could emerge from the capacity of CD15-3 to interact with multiple proteins sharing comparable active site conformations. Guided by the global metabolomics and metabolic network analysis, we analysed the consequences of metabolite abundance differences which are close to nucleotide-metabolism and perturbations elsewhere in the metabolic vicinity. Further, we performed metabolic supplementation experiments with metabolites which showed considerable abundance differences upon CD15-3 treatment to see whether supplementation with these compounds' rescues CD15-3 induced growth inhibition. We observed that supplementation with a subset of metabolites lead to growth recovery with improved lag times and growth rates (Figure 1C and D). We performed recovery experiments by first allowing cells to grow in presence of CD15-3 and then allowing them to grow in normal M9 minimal media. We analysed this data in the context of the metabolic network to narrow down the search options for the plausible CD15-3 non-DHFR cellular target and found maximal perturbation around the folate pathway. The analysis pointed to potential targets upstream of the DHFR catalysed step. We went on to carry out gene over-expression studies for these genes to determine which ones rescue 
growth inhibition by CD15-3. A subset of these overexpressed genes was found to have certain degrees of rescue. Among all the candidate genes folK which codes for HPPK was found to have the highest and very clear complete rescue of growth rate under CD15-3 treatment conditions. Interestingly, an examination of metabolic network analysis combined with structural comparison of folate pathway proteins also predicted HPPK as a plausible CD15-3 target based on the metabolic perturbation signals. Unlike DHFR overexpression which only partially rescued CD15-3 induced growth inhibition, HPPK showed clear recovery at all concentrations of CD15-3. To verify HPPK as a target of CD15-3 we carried out the in vitro enzyme assay with HPPK. We verified that CD15-3 inhibits HPPK activity and has an $\mathrm{IC}_{50}$ of $36.46 \mu \mathrm{M}$. In the Supplement Section 1 (Supplementary Information) we have provided a plausible explanation as to why we see a full rescue from CD15-3 induced growth inhibition with HPPK overexpression compared to partial rescue observed with DHFR overexpression.

Thus, guided by untargeted global metabolomics and the metabolic network and using metabolic supplementation, recovery assays and gene overexpression studies, we could identify the potential non-DHFR target of CD15-3. In our previous work we have shown how CD15-3 delays the emergence of resistant phenotypes (Zhang et al., 2020). Our current work shows that CD15-3 interacts with HPPK apart from DHFR inside the bacterial cells. As mentioned earlier CD15-3 interacts with not only WT DHFR but also the TMP resistant DHFR forms. Now with HPPK as the non-DHFR target, CD15-3 can be considered as the multivalent drug which can block and inhibit two molecular targets inside the bacterial cell. Interestingly being an essential protein bacterial HPPK has been an attractive target for designing antibiotics (Shaw et al., 2014). Thus CD15-3 in principle can serve as a lead to a "monotherapy-analog" of "combination therapy" which blocks the emergence of antibiotic resistant phenotypes by interacting with two targets making it difficult for bacteria to escape antibiotic stress. Although the initial CD15-3 design aimed at achieving potency against WT and TMP resistant DHFR mutants, the additional activity against HPPK presents an additional major promise of CD15.3 as an evolution drug lead. We did not find any specific point mutations in either CD15.3 target folA or folK loci in short-term evolution experiment (Zhang et al., 2020). Thus currently we cannot assess which HPPK evolutionary escape variants might emerge under conditions of longer CD15-3 treatment. Future studies could involve comparative evolutionary studies with "evolution-drug" leads like CD15-3 which are monotherapy-analogues of combination therapies and conventional combination therapies in a pursuit to understand how the evolutionary landscape appears under these conditions of selection pressure.

In conclusion, we showed how integrating multiscale systems-level approaches can reveal novel molecular targets of known and emerging lead antibiotics. Systematic exploration of system level approaches to target discovery has potential broad application to novel formulations of evolution drugs as antibacterial or antiviral stressors and beyond.

\section{Material and Methods}




\section{Antibacterial Growth Measurements and IC 50 values}

Bacterial cultures in M9 minimal medium were grown overnight at $37{ }^{\circ} \mathrm{C}$ and were then normalized to an OD of 0.1 using fresh medium. A new normalization to an $\mathrm{OD}=0.1$ was conducted after additional secondary growth for $\sim 4$ hours. Thereafter the M9 medium and six different concentrations of the CD15-3 in the $96-$ well plates (1/5 dilution) were incubated. The incubation of the plates was performed at $37{ }^{\circ} \mathrm{C}$ and the orbital shacking and absorbance measurements at $600 \mathrm{~nm}$ were taken every 30 min during $15 \mathrm{~h}$. Growth rate was calculated using logistic fitting on matlab.

\section{Metabolomics analysis}

Untargeted global metabolomics was performed to understand the global metabolome of the WT treated and control sets under different experimental contexts. In all the experiments cells were grown in M9 minimal media supplemented with $0.8 \mathrm{~g} / \mathrm{L}$ glucose in a $250 \mathrm{~mL}$ flask and temperature of $37^{0} \mathrm{C}$ was maintained. Cells were pelleted with a brief 2 minutes precentrifugation incubation step on dry ice. After pelleting the cell pellets were mixed with $80 \%$ pre-chilled methanol Samples were thereafter vortexed and incubated in dry ice for $10 \mathrm{~min}$ followed by centrifugation at $4{ }^{\circ} \mathrm{C}$ for $10 \mathrm{~min}$ at maximum speed. The supernatant was collected, and the pellet was repeatedly processed by resorting to the above-mentioned procedures. Samples were stored at $-80^{\circ} \mathrm{C}$ until analyzed by mass spectrometry. In all our experiments at least three independent biological replicates were analyzed. LC-MS analysis in the positive and negative mode was performed as previously described (Bhattacharyya et al., 2016). A list of 48 experimentally measured retention times was used for initial calibration of the retention time predictions. We performed data analysis for untargeted metabolomics using the software the packages MzMatch (Scheltema et al., 2011) and IDEOM (Creek et al., 2012). In untargeted analysis for peak assignment IDEOM include both positive and negative peak M/Z values and predicted retention times calculated based on chemical descriptors. We followed the same method of analysis as we had in one of our earlier studies (Rodrigues and Shakhnovich, 2019).

\section{Recovery Experiments}

WT BW25113 cells were grown both in absence and presence of prospective compound CD15-3 in M9 media supplemented with $0.8 \mathrm{gL}^{-1}$ glucose. Both the naïve (which were not exposed to CD15-3 treatment) and pre-exposed cells (cells treated with CD15-3) were subjected to growth in normal M9 media and growth profiles were analyzed. The entire pre-exponential stage (lag phase) was grouped into equalt time frames and both naïve and pre-exposed cells were harvested for metabolomic studies. Same process was also executed for harvesting both naïve and preexposed cells at late log phase.

\section{Overexpression Experiments}

WT cells were transformed with blank vector plasmids (without inserts) as well as plasmids overexpressing genes viz. thyA, glyA, metF, purH, purC,folD, purD, adk and folk. The genes were under $\mathrm{pBAD}$-promoter and the overexpression of the genes was induced using externally supplemented arabinose $(0.1 \%)$. 


\section{Reporter metabolites analysis of metabolomics data}

To analyze hotspots of metabolite concentration change, we utilized the reporter metabolites algorithm (Patil and Nielsen, 2005) implemented in MATLAB in the COBRA toolbox version 3.0 (Heirendt et al., 2019) and the latest metabolic network reconstruction of E. coli, termed iML1515 (Monk et al., 2017). The reporter metabolites method was modified to utilize metabolite concentration data as an input rather than gene expression data by transposing the stoichiometric matrix of the metabolic model. Default options were used for the reporterMets function. The resulting scores were reported for each reaction in the model.

\section{Protein structural comparison}

Proteins were selected that were nearby in metabolic maps in the pathway of interest as well as some unrelated metabolic proteins. Isomif was used to pull different binding clefts from each of the selected proteins and the 5 largest of each protein binding clefts were kept. The similarity (Tanimoto coefficient) between the clefts of the folA protein structure (PDB ID: 1DRA) and each of the selected clefts of the proteins using isomif which was then plotted to view which binding clefts were most similar. FATCAT was used to compare the overall structural similarity between all of the selected proteins and the results were plotted using a clustermap to look for similar proteins. Visualizations of the proteins were created using nglview.

\section{Statistical and Python plots:}

Statistical analyses of the data and their representation was carried out using Origin pro 8.1 package. Metabolomics data were processed using R based MS converting operation and IDEOM tool. For calibrating $\mathrm{M} / \mathrm{Z}$ (mass to charge) values and retention times of the standard metabolites, XCalibur package was used. For the quantitative depictions of the metabolomics data statistically validated outputs were plotted using Python libraries of matplotlib and seaborn.

\section{HPPK activity assay}

HPPK activity assay was conducted with the help of KinaseGlo ${ }^{\mathrm{TM}}$ assay kit. In this assay, firefly luciferase utilizes the ATP remaining after HPPK catalysis to produce a luminescence signal that is directly proportional to ATP concentration; from this, the HPPK activity can be derived. The enzyme activity calculation and selection of optimum concentration was done following previously published methods (Chhabra et al., 2012). For kinetic measurements, an optimized HPPK concentration of $7 \mathrm{ng} / 50 \mu \mathrm{L}$ assay volume was determined, which allowed for monitoring the first $10 \%$ of reactions turnover in a reasonable assay time period $(20 \mathrm{~min})$.

Measurements were performed in 96-well plates using assay buffer (100 mMTris-HCl/10 mM $\mathrm{MgCl}_{2}, \mathrm{pH} 8.5,0.01 \%$ (w/v) BSA, 0.01\% (v/v) Tween 20 and $10 \mathrm{mM} \beta$-mercaptoethanol). Typically, $5 \mu$ l of test compound (dissolved in 50\% DMSO) and $20 \mu 1$ of enzyme were added to each well followed by $25 \mu \mathrm{l}$ of assay buffer giving $0.3 \mu \mathrm{M}$ pterin and $0.2 \mu \mathrm{M}$ ATP in a total reaction volume $50 \mu \mathrm{l}$. After a 20 minute incubation at room temperature, the enzymatic reaction was stopped with $50 \mu \mathrm{l}$ of KinaseGlo ${ }^{\mathrm{TM}}$ reagent. Luminescence was recorded after a further 10 min using the plate reader (Tecan Infinite M200 Pro). 


\section{Differential interference contrast (DIC)}

WT cells were grown in M9 media supplemented with $0.8 \mathrm{gL}^{-1}$ glucose and casamino acids (mixtures of all amino acids except tryptophan) in absence and presence of CD15-3 at $42^{\circ} \mathrm{C}$ for incubation and $300 \mathrm{rpm}$ constant shaking. Drop in DHFR activity has been associated with cellular filamentation and similar phenotype is observed under TMP treatment(Bhattacharyya et al., 2021). Since CD15-3 targets intracellular DHFR and soluble fraction of cellular DHFR is lower at 42 degrees $\mathrm{C}$ we chose this temperature for our imaging studies (Bershtein et al., 2012).

Aliquots were taken from the growing culture and they were drop casted on agar bed/blocks. These blocks were taken further processed for differential inference contrast (DIC) imaging using Zeis Discovery imaging workstation. Multiple fields were observed and scanned for a single condition type and a minimum of three replicates were used for imaging studies. Similar methods for imaging were used for WT cell types overexpressing folK under conditions of absence and presence of CD15-3 compound. Intellesis Module was used for analyzing DIC images. On average, around 500 cells were analyzed for computing cell length. E. coli cell lengths in our imaging studies were not normally distributed. Nonparametric Mann-Whitney test was therefore used to determine if the cell length distributions were significantly different upon CD15-3 treatment. 


\section{References:}

Ahmad, S., Kirk, S., and Eisenstark, A. (1998). Thymine metabolism and thymineless death in prokaryotes and eukaryotes. Annual review of microbiology 52, 591-625.

Bermingham, A., and Derrick, J.P. (2002). The folic acid biosynthesis pathway in bacteria: evaluation of potential for antibacterial drug discovery. Bioessays 24, 637-648.

Bershtein, S., Choi, J.M., Bhattacharyya, S., Budnik, B., and Shakhnovich, E. (2015). Systems-level response to point mutations in a core metabolic enzyme modulates genotype-phenotype relationship. Cell reports 11, 645-656.

Bershtein, S., Mu, W., and Shakhnovich, E.I. (2012). Soluble oligomerization provides a beneficial fitness effect on destabilizing mutations. Proceedings of the National Academy of Sciences 109, 4857-4862. Bhattacharyya, S., Bershtein, S., Adkar, B.V., Woodard, J., and Shakhnovich, E.I. (2021). Metabolic response to point mutations reveals principles of modulation of in vivo enzyme activity and phenotype. Molecular systems biology in press, 738823.

Bhattacharyya, S., Bershtein, S., Yan, J., Argun, T., Gilson, A.I., Trauger, S.A., and Shakhnovich, E.I. (2016). Transient protein-protein interactions perturb E. coli metabolome and cause gene dosage toxicity. Elife 5, e20309.

Butler, M.S., Blaskovich, M.A., and Cooper, M.A. (2013). Antibiotics in the clinical pipeline in 2013. The Journal of antibiotics 66, 571-591.

Chhabra, S., Barlow, N., Dolezal, O., Hattarki, M.K., Newman, J., Peat, T.S., Graham, B., and Swarbrick, J.D. (2013). Exploring the chemical space around 8-mercaptoguanine as a route to new inhibitors of the folate biosynthesis enzyme HPPK. PloS one 8, e59535.

Chhabra, S., Dolezal, O., Collins, B.M., Newman, J., Simpson, J.S., Macreadie, I.G., Fernley, R., Peat, T.S., and Swarbrick, J.D. (2012). Structure of S. aureus HPPK and the discovery of a new substrate site inhibitor. PloS one 7, e29444.

Creek, D.J., Jankevics, A., Burgess, K.E., Breitling, R., and Barrett, M.P. (2012). IDEOM: an Excel interface for analysis of LC-MS-based metabolomics data. Bioinformatics 28, 1048-1049.

Fridman, O., Goldberg, A., Ronin, I., Shoresh, N., and Balaban, N.Q. (2014). Optimization of lag time underlies antibiotic tolerance in evolved bacterial populations. Nature 513, 418-421.

Heirendt, L., Arreckx, S., Pfau, T., Mendoza, S.N., Richelle, A., Heinken, A., Haraldsdóttir, H.S., Wachowiak, J., Keating, S.M., and Vlasov, V. (2019). Creation and analysis of biochemical constraintbased models using the COBRA Toolbox v. 3.0. Nature protocols 14, 639-702.

Huovinen, P. (1987). Trimethoprim resistance. Antimicrobial agents and chemotherapy 31, 1451. Justice, S.S., Hunstad, D.A., Cegelski, L., and Hultgren, S.J. (2008). Morphological plasticity as a bacterial survival strategy. Nature Reviews Microbiology 6, 162-168.

Li, Z., Jaroszewski, L., Iyer, M., Sedova, M., and Godzik, A. (2020). FATCAT 2.0: towards a better understanding of the structural diversity of proteins. Nucleic Acids Research.

Monk, J.M., Lloyd, C.J., Brunk, E., Mih, N., Sastry, A., King, Z., Takeuchi, R., Nomura, W., Zhang, Z., and Mori, H. (2017). i ML1515, a knowledgebase that computes Escherichia coli traits. Nature biotechnology 35, 904-908.

Olivares Pacheco, J.A., Bernardini, A., Garcia-Leon, G., Corona, F., Sanchez, M.B., and Martinez, J.L. (2013). The intrinsic resistome of bacterial pathogens. Frontiers in microbiology 4, 103.

Patil, K.R., and Nielsen, J. (2005). Uncovering transcriptional regulation of metabolism by using metabolic network topology. Proceedings of the national academy of sciences 102, 2685-2689. 
Rabinowitz, J., Purdy, J., Vastag, L., Shenk, T., and Koyuncu, E. (2011). Metabolomics in drug target discovery. Paper presented at: Cold Spring Harbor symposia on quantitative biology (Cold Spring Harbor Laboratory Press).

Rodrigues, J.V., and Shakhnovich, E.I. (2019). Adaptation to mutational inactivation of an essential gene converges to an accessible suboptimal fitness peak. Elife 8, e50509.

Sangurdekar, D.P., Zhang, Z., and Khodursky, A.B. (2011). The association of DNA damage response and nucleotide level modulation with the antibacterial mechanism of the anti-folate drug trimethoprim. BMC genomics 12, 583.

Scheltema, R.A., Jankevics, A., Jansen, R.C., Swertz, M.A., and Breitling, R. (2011). PeakML/mzMatch: a file format, Java library, $\mathrm{R}$ library, and tool-chain for mass spectrometry data analysis. Analytical chemistry 83, 2786-2793.

Shaw, G.X., Li, Y., Shi, G., Wu, Y., Cherry, S., Needle, D., Zhang, D., Tropea, J.E., Waugh, D.S., and Yan, H. (2014). Structural enzymology and inhibition of the bi-functional folate pathway enzyme HPPK-DHPS from the biowarfare agent $F$ rancisella tularensis. The FEBS journal 281, 4123-4137.

Shimizu, K. (2014). Regulation systems of bacteria such as Escherichia coli in response to nutrient limitation and environmental stresses. Metabolites 4, 1-35.

Silver, L.L. (2007). Multi-targeting by monotherapeutic antibacterials. Nature Reviews Drug Discovery 6, 41-55.

Sullivan, G.J., Delgado, N.N., Maharjan, R., and Cain, A.K. (2020). How antibiotics work together: Molecular mechanisms behind combination therapy. Current Opinion in Microbiology 57, 31-40. Tanega, C., Shen, M., Mott, B.T., Thomas, C.J., MacArthur, R., Inglese, J., and Auld, D.S. (2009). Comparison of bioluminescent kinase assays using substrate depletion and product formation. Assay and drug development technologies 7, 606-614.

Tyers, M., and Wright, G.D. (2019). Drug combinations: a strategy to extend the life of antibiotics in the 21st century. Nature Reviews Microbiology 17, 141-155.

Vincent, I.M., Ehmann, D.E., Mills, S.D., Perros, M., and Barrett, M.P. (2016). Untargeted metabolomics to ascertain antibiotic modes of action. Antimicrobial agents and chemotherapy 60, 2281-2291. Wu, C., Kim, H.K., van Wezel, G.P., and Choi, Y.H. (2015). Metabolomics in the natural products field-a gateway to novel antibiotics. Drug Discovery Today: Technologies 13, 11-17.

Zampieri, M., Zimmermann, M., Claassen, M., and Sauer, U. (2017). Nontargeted metabolomics reveals the multilevel response to antibiotic perturbations. Cell reports 19, 1214-1228.

Zaritsky, A., Woldringh, C.L., Einav, M., and Alexeeva, S. (2006). Use of thymine limitation and thymine starvation to study bacterial physiology and cytology. Journal of bacteriology 188, 1667-1679.

Zhang, Y., Choudhury, S., Rodrigues, J., and Shakhnovich, E. (2020). Development of antibacterial compounds that block evolutionary pathways to resistance. arXiv preprint arXiv:201101098. 


\section{Supporting Information:}

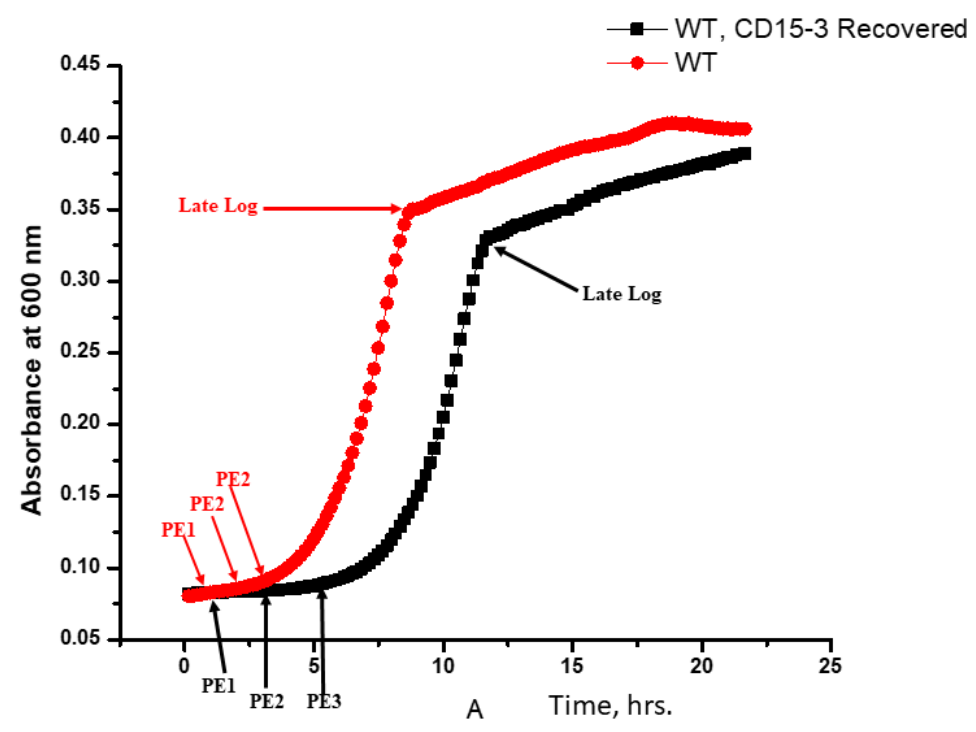

Supplementary Figure 1: Growth curves for WT (naïve in red) and WT pre-exposed cells (CD15.3 recovered in black). PE1, PE2 and PE3 are respectively pre-exponential stages 1, 2 and 3 of growth curve. Stages (PE1, PE2, PE3 and Late Log) marked in red are for naïve WT cells and those marked in black refer to pre-exposed WT. For the recovery assay experiments, naïve and CD15.3 pre-exposed cells were harvested at their respective pre-exponential and late log phases of growth. 

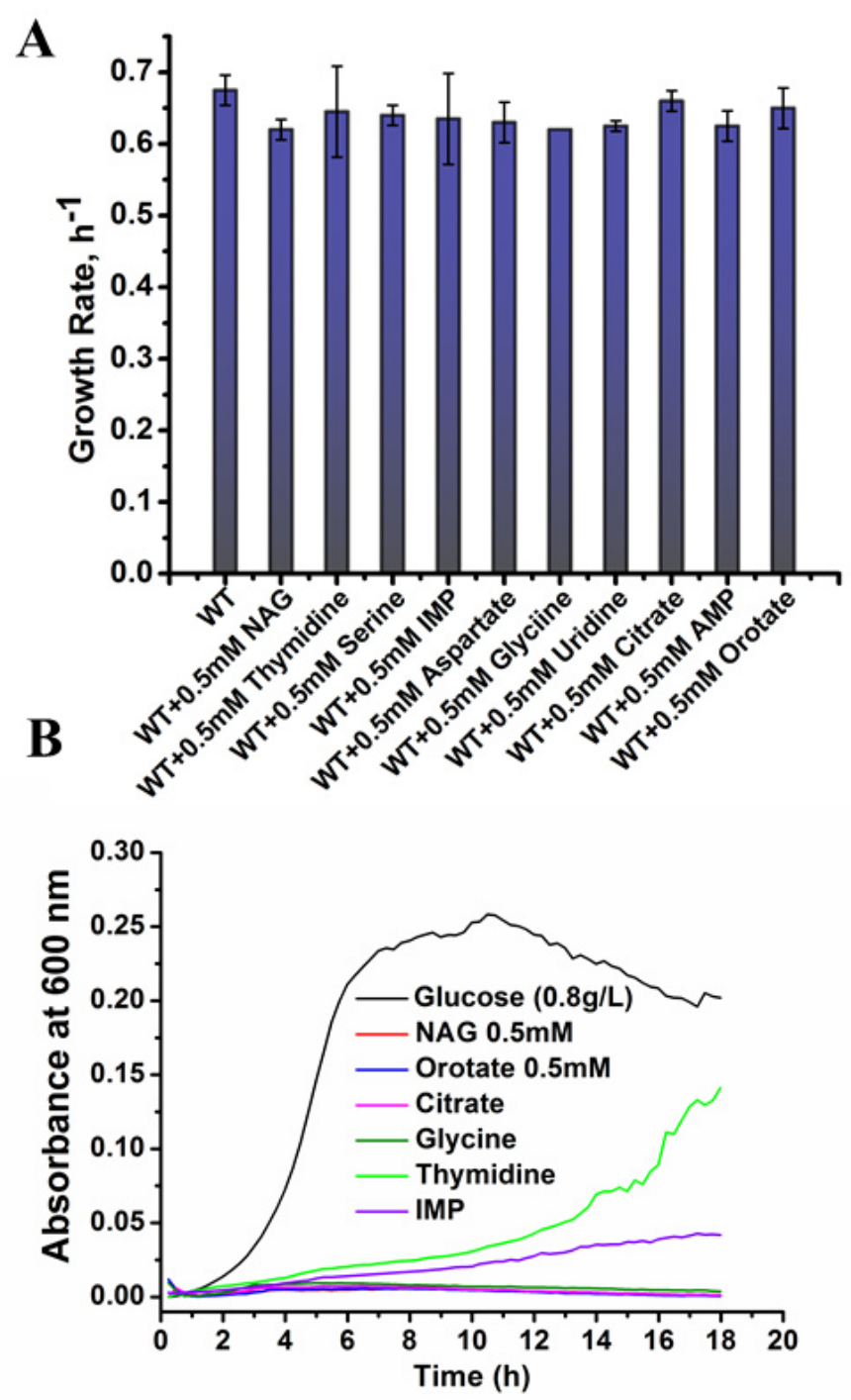

Supplementary Figure 2: Metabolites selected for external supplementation experiments did not show any toxic effect on the growth profiles of the cells. (A) Bar plot showing growth rates of WT cells grown in the presence of metabolites as external supplements in M9 media. (B) Growth curve shows that these external metabolites did not show any potential to function as an alternate $\mathrm{C}$-source in the growth media. 

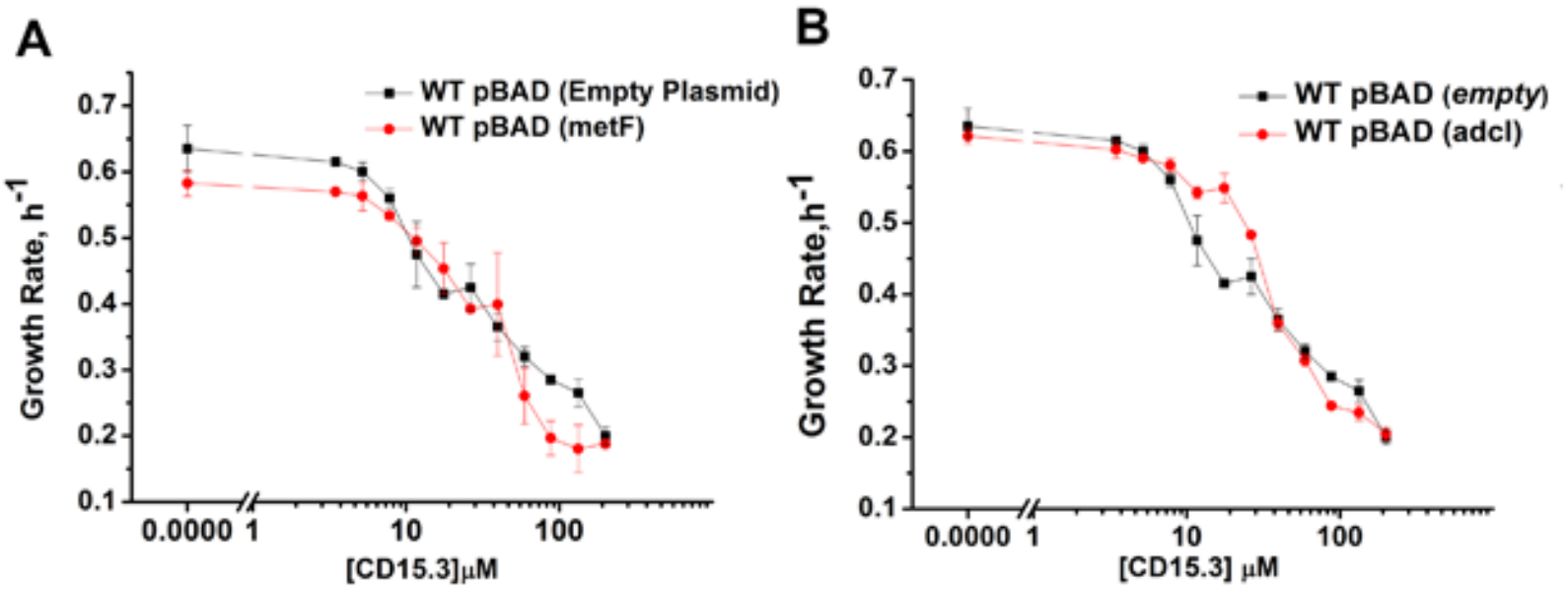

Supplementary Figure 3: (A) Overexpression of metF (encoding MTHFR) under pBAD promoter with $0.1 \%$ arabinose induction did not lead to recovery from CD15.3 induced growth inhibition. (B) Overexpression of ADCL (encoded by gene pabC) under pBAD promoter with $0.1 \%$ arabinose induction was found to have no recovery effect on CD15.3 treated cells. 


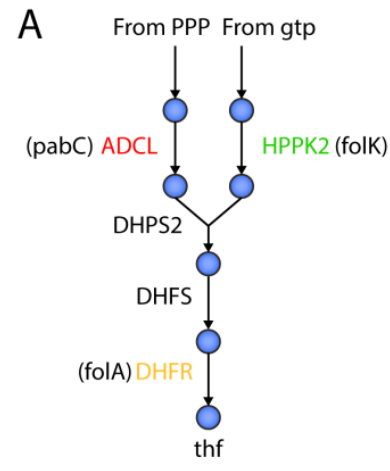

Folate Biosynthesis

D

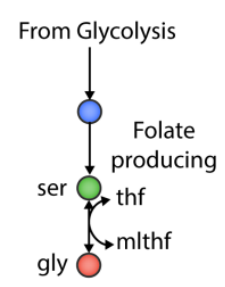

Serine Metabolism

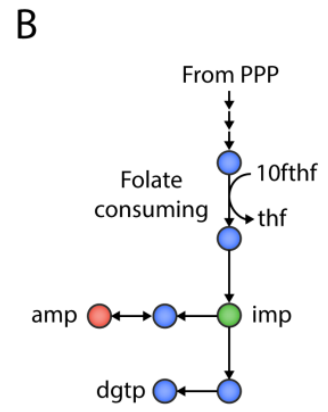

Purine Biosynthesis

E

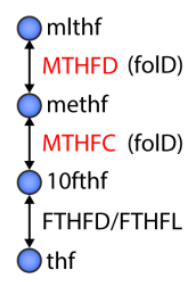

Folate Interconversion
C From glutamate

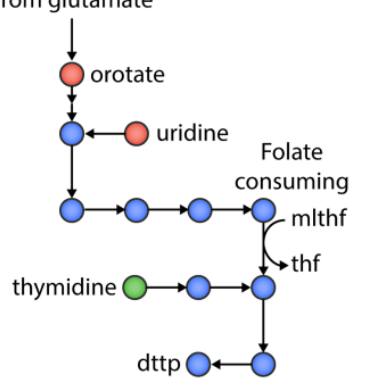

Pyrimidine Biosynthesis

Does not rescue growth

Rescues growth

Enzyme Does not rescue growth

Enzyme Partially rescues growth

Enzyme Rescues growth

\section{Supplementary Figure 4: Summary of selected supplement and overexpression evidence for} a folate-related mechanism of action of CD15. A) De novo folate biosynthesis. Three enzymes were overexpressed. Overexpression of the intended targeted, folA, partially restored growth. Overexpression of pabC (adcl) did not restore growth. B) Purine biosynthesis. IMP and AMP metabolic supplementation was done. IMP supplementation rescued growth while AMP had no effect in rescuing CD15.3 induced growth inhibition. C) Pyrimidine biosynthesis. Thymidine supplementation led to growth rescue from CD15.3 induced inhibition and Orotate and uridine had no effect in recovery. D) Serine biosynthesis. Metabolic supplementation of serine resulted in growth rescue. E) Folate interconversion. 


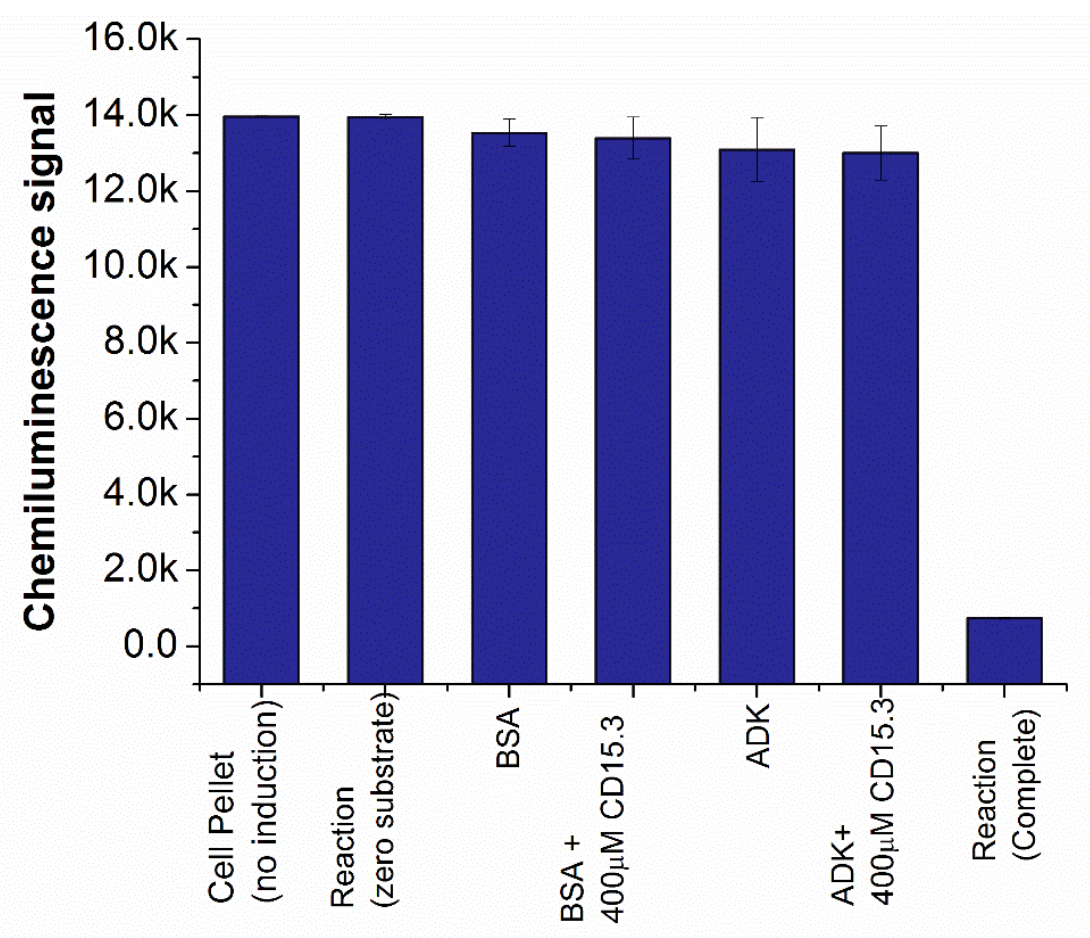

Supplementary Figure 5: Bar plot showing the range of chemiluminescence signal at zero substrate (no reaction) condition and the end of reaction. Absolute chemiluminescence signal intensity as observed under different control conditions. 1000 fold diluted cell pellets (same as is used for folk overexpressing cells) obtained from cells not induced with $0.1 \%$ arabinose did not lead to any drop in the chemiluminescence signal. Similar readout was also found with BSA (a non-ATP using protein) and Adk (an ATP dependent protein) in presence of CD15-3 in the assay buffer.

Supplementary Text: The case when 2 enzymes are inhibited by a common inhibitor.

Here we consider the situation when two enzymes are inhibited by common inhibitor. We use the following notations: 
$x_{0}$ total concentration of the inhibitor (i.e. free + bound)

$x_{\text {free }}$ concentration of free inhibitor in solution

$E_{1}^{0}$ total concentration of enzyme 1 in the cell

$E_{1}^{\text {free }}$ concentration of free (unbound) enzyme 1 in the cell

$\left[E_{1} x\right]$ concentration of bound enzyme 1-inhibitor complex in the cell

$E_{2}^{0}$ total concentration of enzyme 2 in the cell

$E_{2}^{\text {free }}$ concentration of free (unbound) enzyme 2 in the cell

$\left[E_{2} x\right]$ concentration of bound enzyme 2-inhibitor comnplex in the cell

$\mathrm{K}_{d 1}, \mathrm{~K}_{d 2}$ binding affinities of enzyme 1 and 2 respectively to the inhibitor

Using Law of Mass Action and conservation of mass for all three components (enzymes 1 and 2 and the inhibitor) we get for both enzymes:

$$
\begin{aligned}
& {\left[E_{1} x\right]=\frac{E_{1}^{\text {free }} x_{\text {free }}}{K_{d 1}}} \\
& {\left[E_{2} x\right]=\frac{E_{2}^{\text {free }} x_{\text {free }}}{K_{d 2}}} \\
& {\left[E_{1} x\right]+\left[E_{2} x\right]+x_{\text {free }}=x_{0}} \\
& {\left[E_{1} x\right]+E_{1}^{\text {free }}=E_{1}^{0}} \\
& {\left[E_{2} x\right]+E_{2}^{\text {free }}=E_{2}^{0}} \\
& E_{1}^{0}-E_{1}^{\text {free }}=\frac{E_{1}^{\text {free }} x_{\text {free }}}{K_{d 1}} ; E_{1}^{\text {free }}=\frac{E_{1}^{0}}{1+\frac{x_{\text {free }}}{K_{d 1}}} ;\left[E_{1} x\right]=\frac{E_{1}^{0} x_{\text {free }}}{x_{\text {free }}+K_{d 1}} \\
& E_{2}^{0}-E_{2}^{\text {free }}=\frac{E_{2}^{\text {free }} x_{\text {free }}}{K_{d 2}} ; E_{2}^{\text {free }}=\frac{E_{2}^{0}}{1+\frac{x_{\text {free }}}{K_{d 2}}} ;\left[E_{2} x\right]=\frac{E_{2}^{0} x_{\text {free }}}{x_{\text {free }}+K_{d 2}} \\
& \frac{E_{1}^{0} x_{\text {free }}}{x_{\text {free }}+K_{d 1}}+\frac{E_{2}^{0} x_{\text {free }}}{x_{\text {free }}+K_{d 2}}+x_{\text {free }}=x^{0} \\
& x_{\text {free }}^{3}+x_{\text {free }}^{2}\left(E_{1}^{0}+E_{2}^{0}+K_{d 1}+K_{d 2}-x_{0}\right)+x_{\text {free }}\left(E_{1}^{0} K_{d 2}+E_{2}^{0} K_{d 1}+K_{d 1} K_{d 2}-x_{0}\left(K_{d 1}+K_{d 2}\right)\right)-x_{0} K_{d 1} K_{d 2}=0
\end{aligned}
$$

Now consider the overexpression case $E_{1}^{0} \gg E_{2}^{0} ; K_{d 1} \approx K_{d 2}$ i.e. $\quad \frac{E_{1}^{0} x_{\text {free }}}{x_{\text {free }}+K_{d 1}} \gg \frac{E_{2}^{0} x_{\text {free }}}{x_{\text {free }}+K_{d 2}}$ 
This inequality means that most of the inhibitor is bound to enzyme 1 (naturally as it is overexpressed). In this case the cubic equation for free concentration becomes quadratic because we neglect in the first approximation the inhibitor bound to enzyme 2

$$
\begin{aligned}
& E_{1}^{0} x_{\text {free }}+x_{\text {free }}{ }^{2}+K_{d 1} x_{\text {free }}=x_{0} x_{\text {free }}+K_{d 1} x_{0} \\
& x_{\text {free }}{ }^{2}+x_{\text {free }}\left(E_{1}^{0}+K_{d 1}-x_{0}\right)-K_{d 1} x_{0}=0 \\
& x_{\text {free }}=\frac{-\left(E_{1}^{0}+K_{d 1}-x_{0}\right)+\sqrt{\left(E_{1}^{0}+K_{d 1}-x_{0}\right)^{2}+4 K_{d 1} x_{0}}}{2}
\end{aligned}
$$

assume high overexpression

$$
\begin{aligned}
& E_{1}^{0} \gg x_{0}, K_{d 1} \\
& x_{\text {free }} \approx \frac{x_{0} K_{d 1}}{E_{1}^{0}} \\
& E_{2}^{\text {free }}=\frac{E_{2}^{0}}{1+\frac{x_{0}}{E_{1}^{0}} \frac{K_{d 1}}{K_{d 2}}}
\end{aligned}
$$

Thus the effect of overexpression of one enzyme affects the way how another enzyme is inhibited by the same inhibitor in the positive way: the more one enzyme is overexpressed the less other enzyme is inhibited because the inhibitor is sequestered into a binding complex with the overexpressed enzyme. However, with respect to increased expression of the inhibitor we can see 2 regimes:

when overexpression is high $\frac{K_{d 2} x_{0}}{K_{d 1} E_{1}^{0}} \ll 1$.

In this case $E_{2}^{\text {free }}=\frac{E_{2}^{0}}{1+\frac{x_{0} K_{d 1}}{E_{1}^{0} K_{d 2}}} \approx E_{2}^{0}\left(1-\frac{x_{0}}{E_{1}^{0}} \frac{K_{d 1}}{K_{d 2}}\right) \approx E_{2}^{0}$

i.e. fitness does not depend on the inhibitor concentration because the overexpressed enzyme sequesters most of the inhibitor and the other target enzyme can function uninhibited.

In the opposite case of high concentration of the inhibitor relatively to the overexpressed enzyme or when binding affinities differ strongly between two enzymes, residual inhibitor can inhibit second (not overexpressed) enzyme. 
This scenario corresponds to the case $\frac{K_{d 2} x_{0}}{K_{d 1} E_{1}^{0}} \gg 1$ i.e. when there is plenty of inhibitor - more than overexpressed enzyme can bind - the approximation is different and the result is

$$
\begin{aligned}
& x_{\text {free }} \approx x_{0}-E_{1}^{0} \\
& E_{2}^{\text {free }}=\frac{E_{2}^{0}}{1+\frac{x_{0}-E_{1}^{0}}{K_{d 2}}} \approx \frac{E_{2}^{0}}{\frac{x_{0}-E_{1}^{0}}{K_{d 2}}}
\end{aligned}
$$

We conclude that there can be an asymmetry between the effect of overexpression of HPPK and DHFR. If HPPK is highly overexpressed such that it sequesters most of the inhibitor, then fitness - concentration of the inhibitor curve will be flat. If DHFR is less expressed from the plasmid, i.e. at lower concentration of arabinose as in our experiment $(0.005 \%$ for folA vs $0.1 \%$ for HPPK) then upon addition of inhibitor the inhibitor-growth will be flat at lower concentration of the inhibitor followed by loss of fitness at higher concentration of the inhibitor due to inhibition of HPPK by remaining inhibitor. Inhibition of growth occurs in this case when concentration of the inhibitor becomes greater or equal to concentration of the overexpressed protein. E.g. 1000 fold overexpression of DHFR equals to about 100 micromoles concentration in the cell - roughly similar to concentration of CD15.3 in the experiments. 